\title{
Conformal higher-order remeshing schemes for implicitly defined interface problems
}

\author{
S. Omerović and T.P. Fries \\ Institute of Structural Analysis, Graz University of Technology, Lessingstr. 25/II, 8055 Graz, Austria
}

\begin{abstract}
A new higher-order accurate method is proposed that combines the advantages of the classical $p$-version of the FEM on body-fitted meshes with embedded domain methods. A background mesh composed by higher-order Lagrange elements is used. Boundaries and interfaces are described implicitly by the level set method and are within elements. In the elements cut by the boundaries or interfaces, an automatic decomposition into higher-order accurate sub-elements is realized. Therefore, the zero level sets are detected and meshed in a first step which is called reconstruction. Then, based on the topological situation in the cut element, higher-order sub-elements are mapped to the two sides of the boundary or interface. The quality of the reconstruction and the mapping largely determines the properties of the resulting, automatically generated conforming mesh. It is found that optimal convergence rates are possible although the resulting sub-elements are not always well-shaped.
\end{abstract}

Keywords: p-FEM, higher-order element, level-set method, fictitious domain method, embedded domain method, XFEM, interface problem

\section{Introduction}

Numerical simulations in all fields of computational mechanics often involve complex-shaped, possibly curved domains and/or interfaces with arbitrary geometry. Examples and applications are numerous and include e.g. in solid mechanics, the modelling of composite materials with complex microstructure [37], material inhomogeneities [52], solidification [14] and topology optimization [7, 56]. Further applications can be found in fluid mechanics and include interfaces in multi-phase flows [24] or problems in multi-physics. Such applications feature non-smooth solutions and involve differential equations with discontinuities in the coefficients [1] or even different PDEs across the interfaces. It is well-known that the accuracy of FEM approximations is severely degraded if meshes are used with elements that do not conform to the boundaries and interfaces.

Therefore, over the years a lot of attention has been drawn to the development of techniques to handle interface problems. Several different approaches have been proposed and in terms of mesh representation, the methods can roughly be divided into two different categories. The first category consists of body fitted finite element methods, where all interfaces and boundaries align with element edges. The second category involves the large spectrum of embedding domain methods or fictitious domain methods that use a non-conforming (background) mesh and enforce boundary and interface conditions by tailored approaches, e.g. Lagrange multipliers, penalty methods or Nitsche's method.

In body fitted finite element methods, the computational meshes are usually generated in two different ways. The first approach is to create a mesh with straight edges and then transform this linear discretization into higherorder elements, see [49, 48, 36, 35]. The other possibility is to construct a higher-order mesh directly by creating curved boundaries and using them to discretize the interior of the domain, see [49]. Because the interface can be arbitrarily complex, curved or even in motion, the generation of interface-conforming meshes can be very costly from a computational point of view. Even worse, a large amount of human interaction may be required to generate suitable meshes, in particular with higher-order elements. However, the outstanding advantage is that once a conforming mesh is created, basically any finite element solver based on the isoparametric concept can process it. No specialized 
treatment for the boundary conditions is needed and standard Gaussian quadrature rules are used for the numerical integration of the weak form. All these advantages are also present in the approach proposed herein.

The second category, i.e. embedding domain methods, often uses a structured background mesh as a starting point, which does not account for the element boundaries. The location of the interfaces may, for example, be described via the level set method, see [41, 40, 6]. Methods characterized as embedding domain methods are e.g. the immersed boundary method [43, 16], cut finite element method [10, 11, 9, 27], finite cell method [42, 18, 46, 45], unfitted finite element methods [4, 31] and the parametric finite element method [20]. Even the extended finite element method (XFEM) can be cast in the realm of embedding domain methods for some specific applications, see e.g [23], or in a higher order context [12, 30]. In all these methods, the boundaries or interfaces are within elements so that the application of boundary and interface conditions as well as the numerical integration in cut elements is quite involved. Furthermore, the conditioning of the system of equations may be degraded due to largely different supports of the involved shape functions.

Herein, based on the method introduced in [39] and [28] in a linear, two-dimensional context, we propose a higher-order accurate method which is located somewhere in-between the two aforementioned methodologies. The method uses a background mesh composed by higher-order elements; the mesh does not have to be Cartesian nor structured. Elements that are cut by the implicitly defined boundaries and interfaces are automatically decomposed into conforming sub-elements. This decomposition is based on a two-step procedure where, firstly, the zero levels of level set functions are identified and meshed. Then, secondly, tailored mappings are introduced which define conforming, higher-order sub-elements on the two sides of the interface. Due to the similarities to the low-order method proposed in [39], the introduced approach is labeled a higher-order conformal decomposition finite element method (CDFEM). Similar approaches that follow from mesh subdivision and mesh adaptation are sometimes labelled cut-cell method are found in the context of higher-order discontinuous Galerkin methods (DG) in [19].

The organization of this paper is as follows. Section 2 describes some model problems and gives a short overview of the proposed method. Section 3 shows how to identify and discretize the approximated zero level set by means of higher-order one-dimensional interface elements. This is further referred to as reconstruction. The employed elementwise subdivision technique is discussed in detail in Section 4 and valid topologies for triangular and quadrilateral elements are defined. Furthermore coordinate mappings are discussed that define the location of the internal nodes of the generated higher-order sub-elements. Section 5 contains numerical examples illustrating the accuracy and the stability of the proposed method. All results are in excellent agreement with the analytical solutions and exhibit optimal higher-order convergence rates. We conclude in Section 6 and give an overview of open topics as well as an outlook on future work.

\section{Proposed technique in a nutshell}

Let $\Omega$ be a domain embedded in the Euclidean space $\mathbb{R}^{2}$. The domain is bounded by the external interface $\Gamma_{\text {ext }}$. Inside $\Omega$, there may be internal interfaces $\Gamma_{i n t}$ separating, for example, regions with different material properties or the two sides of a crack path. The interfaces $\Gamma_{i n t}$ and $\Gamma_{\text {ext }}$ are sharp and possibly curved.

For the solution of a BVP in such a domain with the classical FEM, it is crucial to construct a conforming mesh in order to achieve optimal convergence properties. Thus, the elements must consider the position of the interfaces, i.e. element edges must align there. Furthermore, no hanging nodes are typically accepted. A mesh for some schematic domain is shown in Fig. 1 (a). It is not surprising that the generation of the mesh and the maintenance for possibly evolving interfaces are not trivial tasks, especially not with higher-order elements..

Therefore, the aim is to use simple higher-order meshes, further on referred as background meshes, which do not have to account for any of the (external and internal) interfaces. These are composed by standard Lagrange elements which may be triangles or quadrilaterals. These background meshes are simple to construct, but the proposed approach is not necessarily restricted to Cartesian nor structured grids. We propose a procedure to automatically decompose elements in the background mesh that are cut by an arbitrary interface into isoparametric, higher-order sub-elements. This leads to meshes as shown in Fig. 1. (b). It will be seen later that approximations with optimal accuracy are possible on these meshes, although the element shapes are often quite awkward. 


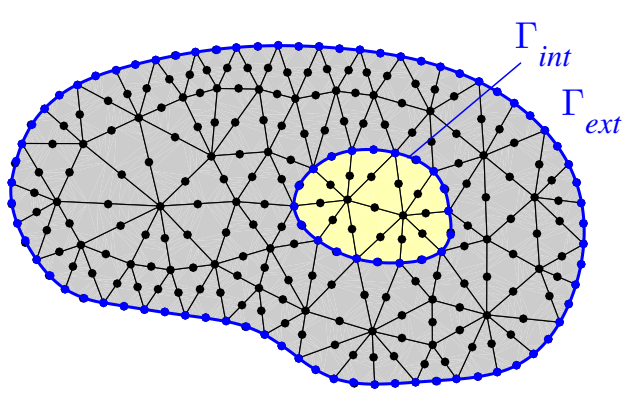

(a) Conforming mesh

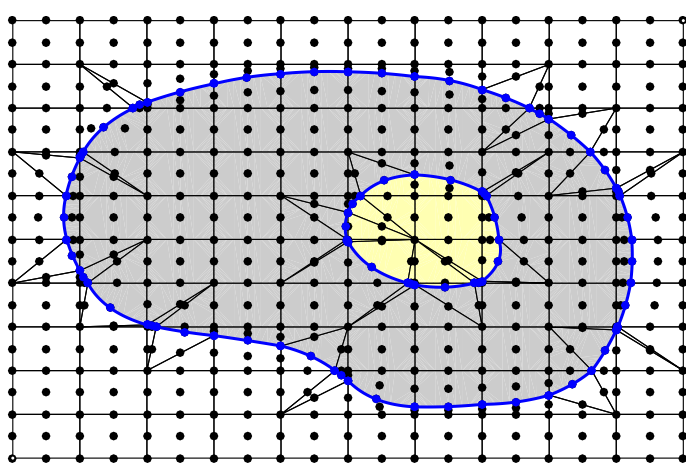

(b) Automatically decomposed background mesh

Figure 1: (a) Classical conforming mesh and (b) the mesh automatically generated from decomposing the background mesh. Both meshes feature higher-order (here: quadratic) Lagrange elements.

Herein, it is assumed that the position of the interfaces are defined by the level set method. That is, a scalar function $\phi(\boldsymbol{x})$ is introduced whose zero level set defines the position of the interfaces. In the present work we restrict ourselves to the case of one level set function which however enables the definition of several unconnected interfaces. The level set values are prescribed at the nodes of the higher-order background mesh and interpolated by the finite element shape functions in between. The discretized level set function is further on referred to as $\phi^{h}$.

The procedure for the automatic decomposition of cut elements is sketched as follows: Following the work of [21], the zero level set of $\phi^{h}$ is first detected and meshed by higher-order interface elements. This is done in the background reference elements as depicted by the blue line in Fig. 2 and is called reconstruction. Here, we moderately modify the procedure to achieve the goals of this work. The second step examines the decomposition into higher-order sub-elements which align with the reconstructed level set. Therefore, customized mappings $\boldsymbol{r}_{Q}(\boldsymbol{a})$ and $\boldsymbol{r}_{T}(\boldsymbol{a})$ for quadrilaterals and triangles, respectively, are introduced from the reference elements to the sub-elements in the cut reference background element. Finally, the reference background element is mapped to the physical domain using the isoparametric mapping, denoted as $\boldsymbol{x}(\boldsymbol{r})$.

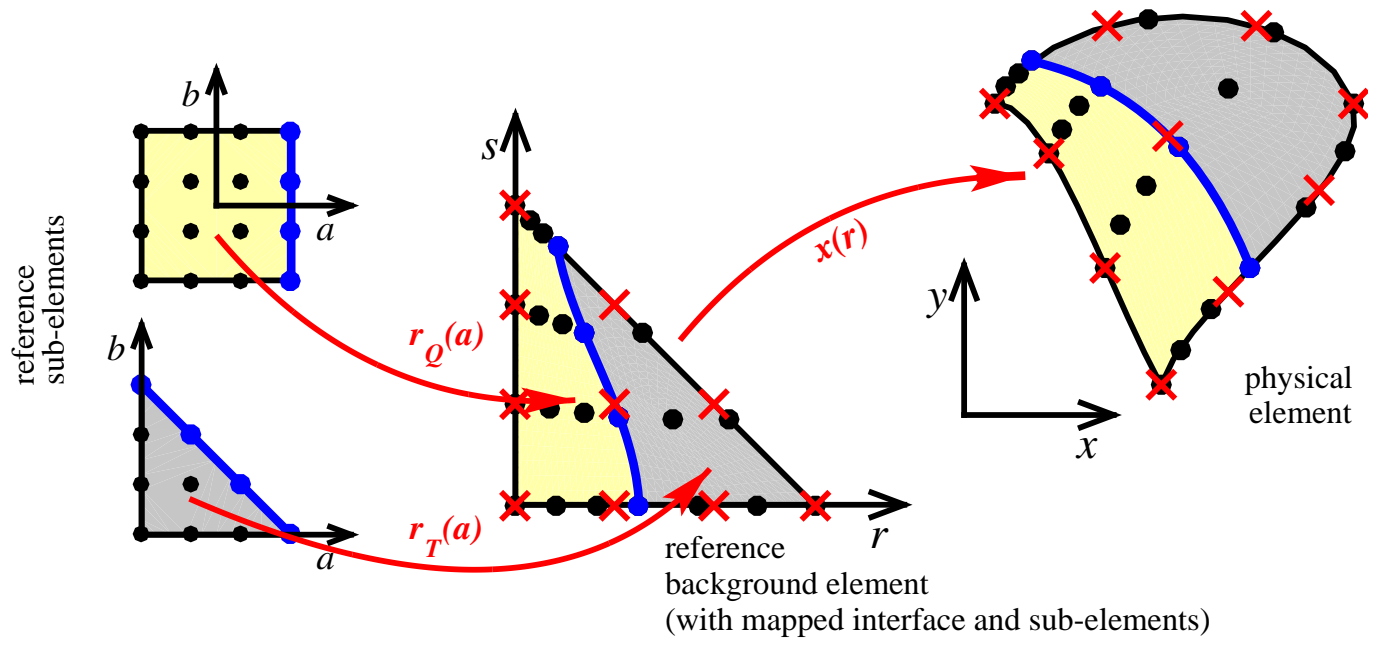

Figure 2: This sketch demonstrates the two mappings (coordinate transformations) that are needed for the proposed remeshing scheme. The mapping $\boldsymbol{x}(\boldsymbol{r})$ is defined by the background element. The mappings $\boldsymbol{r}_{Q}(\boldsymbol{a})$ and $\boldsymbol{r}_{T}(\boldsymbol{a})$ are both based on the interface element. (black dots: nodes of the decomposed sub-elements; red crosses: nodes of the background element; blue dots: nodes of the interface element) 


\section{Reconstruction of the zero level sets}

The aim of this section is to approximate the zero level set of the level set function by means of higher-order finite elements, called interface elements herein. As this paper is restricted to two dimensions, the discretized zero level set is composed by line elements. The topic of reconstructing zero level sets is discussed in more detail in [21], including the extension to the three-dimensional case.

The strategy has a local character, that is, the reconstruction of the zero isoline advances element by element. The choice of Lagrange elements enforces continuity between the elements. The first step is to transfer the level set data, given in the physical domain, to the corresponding reference domain, as the reconstruction is executed in the reference element. This is particularly simple for Lagrange elements as the nodal values $\phi\left(\boldsymbol{x}_{n}\right)$ are taken as nodal values $\phi\left(\boldsymbol{r}_{i}\right)$ in the reference element. Thereby, the indices $n$ and $i$ denote a specific node in global and local element numbering, respectively. Based on this information, elements that are cut by the zero level set are detected. Once the cut elements are identified, it must be ensured that the level set data is valid in the sense that a unique subdivision of the element is possible. For topologically valid cut elements, a scalar root finding algorithm is provided, giving any desired number of points on the interface. These points serve as nodes of the higher-order interface elements approximating the zero level set.

\subsection{Detection of cut elements}

An element is considered as cut, when the data at a finite number of points indicates that the approximated zero level set function $\phi^{h}$ crosses the element domain. To decide whether the element is cut, a sample grid with a user defined resolution is used in the parent element, see Fig. 4. The resolution of this auxiliary grid should depend on the number of element nodes. For the calculations performed in this work three points between element nodes in each dimension were chosen. Using the element functions and the nodal values $\phi^{h}\left(\boldsymbol{r}_{i}\right), i=\{1, . . n\}$, the level set is interpolated at all grid points. The set of grid points assigned to an element is defined as $I_{\text {grid }}$. The check for cut elements is then done by an inspection of the sign of the grid level set values. The considered element is cut if

$$
\min _{i \in I_{\text {grid }}}\left(\phi_{i}\right) \cdot \max _{j \in I_{\text {grid }}}\left(\phi_{j}\right)<0 .
$$

It is important to note that nodal values are not sufficient for the check due to the non-positiveness of the Lagrange shape functions. Hence, a sample grid with suitable resolution is crucial for the check.

\subsection{Valid level set data}

Arbitrary level set data in a higher-order element may result in very complex patterns of zero level sets and the implied regions where $\phi(\boldsymbol{x})$ is positive or negative, respectively. Therefore, it is crucial to impose restrictions on the complexity of the level set data. Herein, we call the level set data in a cut element according to Eq. (1) valid if (i) the element boundary is cut two times and (ii) the zero level set divides the element domain $\Omega_{r}^{h}$ into two sub-domains. However, even valid level set data has to meet some restrictions regarding the curvature of the zero level set, which is discussed in more detail in Section 4

Fig. 3.a) shows valid level set data subdividing the reference element into two sub-domains. Inclusions as seen in Fig. 3 (b) violate the conditions, hence, the level set data is not valid. A situation violating the second condition is shown in Fig. 3.(c). Here the level set data changes more than two times over the element edges, producing more than two separate domains. If there is a high curvature locally, see Fig. 3(d), the level set data is valid according to the conditions above, however, the reconstruction may not be successful anyway (which is automatically detected). If the element is regarded as cut but features invalid level set data, we note that a (locally) refined background mesh always produces valid level set data that meets the conditions from above.

\subsection{Reconstruction of zero level set by Newton-Raphson procedure}

Assuming that the element is cut and the level set data is valid, the task is now to locate element nodes of the interface element with a user-defined order on the zero level set. The process starts with finding two roots on the element boundary. Then, a number of points $\boldsymbol{P}_{i}$, where $i$ depends on the desired element order, are evenly distributed between the two roots on the boundary. These points serve as the starting guesses for the iterative root-finding inside the element. 


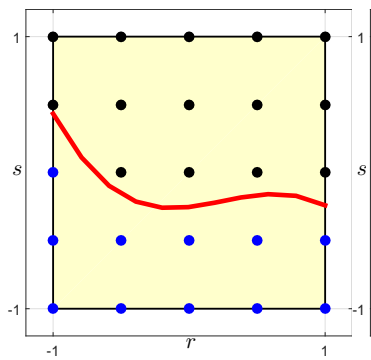

(a)

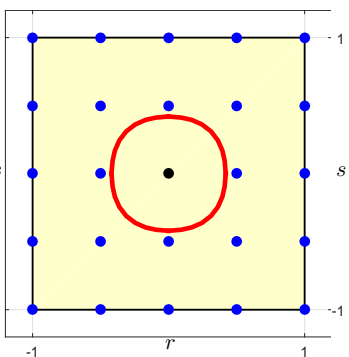

(b)

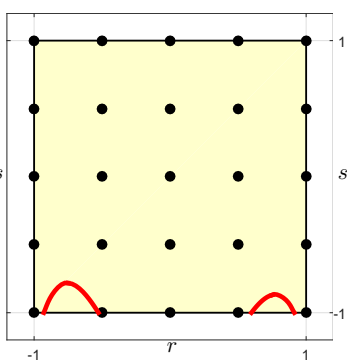

(c)

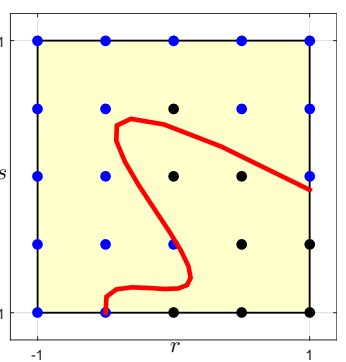

(d)

Figure 3: Zero level sets in reference elements are shown according to (a) valid level set data, (b) and (c) invalid data. (d) results from valid level set data, however the reconstruction is likely to fail.

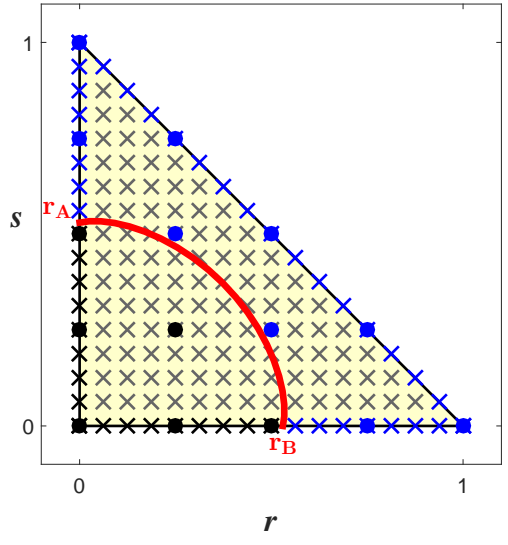

(a) Triangular element

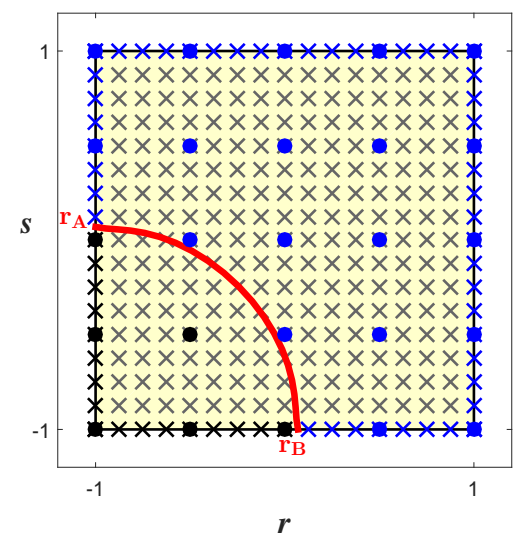

(b) Quadrilateral element

Figure 4: Detection of the intersections of the zero level set with the element boundary based on a sample grid. (blue and black dots: element nodes with positive and negative level-set values, respectively. The crosses build the sample grid)

\subsubsection{Root search on the element boundary}

The procedure starts with finding the two intersections of the zero level set with the element boundary. Therefore, the sample grid from above is considered on the boundary, see Fig. 4. Based on the signs of the level set data in two adjacent grid points, intervals containing zeros of $\phi^{h}$ can be located immediately. The zeros are labeled as $\boldsymbol{r}_{A}$ and $\boldsymbol{r}_{B}$ and are found by applying an iterative root finding algorithm along the edge. The middle of the interval is chosen as a starting guess for the iteration and the algorithm is given by

$$
\boldsymbol{r}^{i+1}=\boldsymbol{r}^{i}-\frac{\phi^{h}(\boldsymbol{r})}{\nabla_{\boldsymbol{r}} \phi^{h}\left(\boldsymbol{r}^{j}\right)}
$$

For all considered topological cases, a cut element edge has either one or two roots, depending on the topological situation. In this section we restrict ourselves to the case of an edge having only one root. As elaborated further in Section 4 this leads to a topological case labeled as local topology, which is the starting point of the subdivision scheme. The configuration with two roots on one element edge, designated as global topology, is also described in Section 4. In this case the approach is different, but for the reconstruction of the zero level set reduces to the same procedure as described here.

\subsubsection{Root search inside an element}

For a detailed discussion concerning the root search inside an element, with different choices of start points and search directions, we refer to [21]. Once the root search along the boundary is done, the two roots are connected 


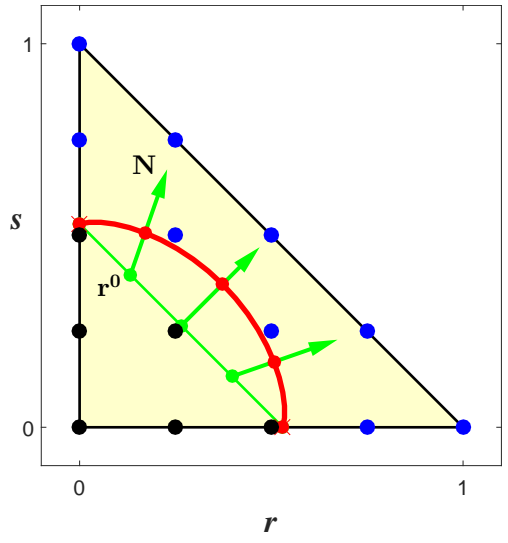

(a)

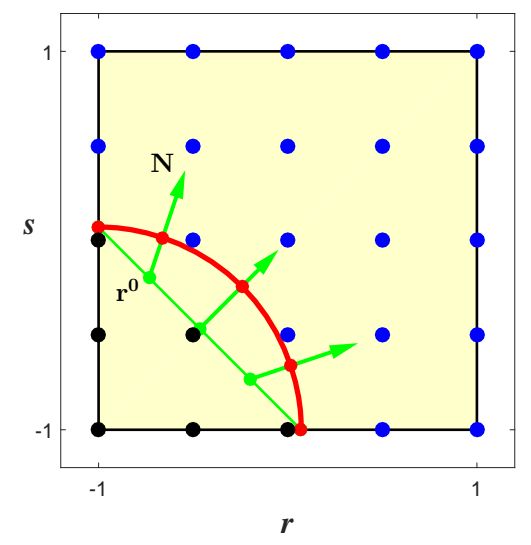

(b)

Figure 5: Search for the roots of $\phi^{h}$ inside the reference element, using a search path specified by the starting point $\boldsymbol{P}=\boldsymbol{r}^{0}$ and the search direction $N$ for a triangular (a) and a quadrilateral (b) element.

by a straight line. On this line, a number of nodes $\boldsymbol{P}_{i}$, associated to the desired order of the interface element are distributed equally spaced. This gives a rough approximation of the zero isoline, and is further referred to as "linear reconstruction", depicted by the green line in Fig. 5. The root search is then executed along a straight path, going through the start point $\boldsymbol{P}=\boldsymbol{r}^{0}$ with a direction $\boldsymbol{N}$ given by the gradient of the level set function at $\boldsymbol{P}$, i.e. $\boldsymbol{N}=\nabla_{\boldsymbol{r}} \phi^{h}(\boldsymbol{P})$. Applying a Newton-Raphson approach along $\boldsymbol{N}$, similar to Eq. 27 yields

$$
\boldsymbol{r}^{i+1}=\boldsymbol{r}^{i}-\frac{\phi^{h}\left(\boldsymbol{r}^{i}\right)}{\nabla_{\boldsymbol{r}} \phi^{h}\left(\boldsymbol{r}^{j}\right) \cdot \boldsymbol{N}} \cdot \boldsymbol{N}
$$

After the residuum $\left|\boldsymbol{r}^{i+1}-\boldsymbol{r}^{i}\right|$ of Eq. 3 falls below a user-specified convergence tolerance (in the performed calculations this tolerance is set as $\approx 10^{-12}$ ) the newly found coordinates of the roots are set as nodal coordinates of the higher-order interface element, see the red line in Fig. 5. This interface element, further on referred to as $\Gamma_{r}^{h}$, will finally serve as a new (curved) edge for higher order triangular or quadrilateral elements on the two sides of the interface. 


\section{Conform remeshing of a background element}

At this point we assume that a general, higher-order cut background element $\Omega_{r}^{h}$ is given and the zero level set is successfully approximated by the reconstructed interface element $\Gamma_{r}^{h}$, see Section 3 . The aim is to: firstly, to identify all topological cases leading to low-order sub-cells with possibly one higher-order side coinciding with $\Gamma_{r}^{h}$ and, secondly, to find a map for the element nodes of higher-order elements into these sub-cells, which is not trivial as seen below. It is noted that the resulting decomposition features triangular and quadrilateral elements.

There are several mappings and transformations involved and Fig. 6 shows the complete process in one diagram. Emanating from the physical element $\Omega_{x}^{h}$, in step 1 the level set values are transferred to the parent domain $\Omega_{r}^{h}$, where the reconstruction of the zero level set is realized. In step 2 the subdivision of $\Omega_{r}^{h}$ takes place. The element is subdivided into a few predefined topologies depending on the cut situation. The only missing link for a working computational mesh is a node distribution within the element patch that preserves optimal accuracy for higher-order elements. This is done by means of a customized mapping procedure, sketched in step 3 of Fig. 6, wherefore the approach for triangular and quadrilateral elements differentiates significantly. Once the nodal coordinates are determined, a conforming higher-order element patch is obtained. At last, in step 4 the data is projected back to the physical domain via an isoparametric mapping.

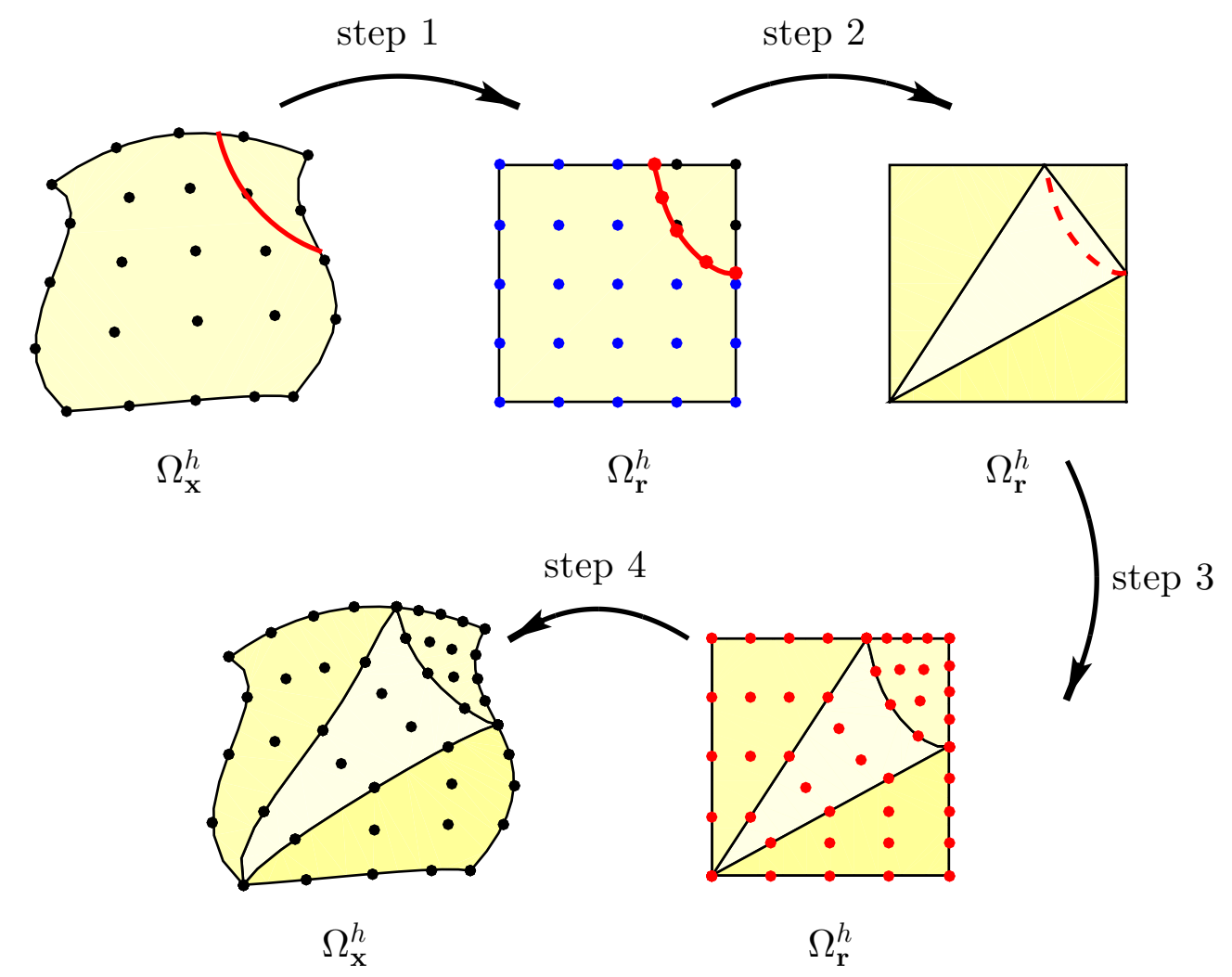

Figure 6: Different steps involved in the remeshing strategy.

Regarding the nomenclature of the following topology classifications, some abbreviations are introduced. The first letter describes the type of element. This can be either $\mathbf{T}$ for triangular or $\mathbf{Q}$ for quadrilateral elements followed by a combination of subscripts and/or a superscripts. The index of the subscripts gives the information which edges are cut, whereas the superscripts describes which nodes are cut. So $\mathbf{Q}_{I J}$ denotes a quadrilateral, with a zero level set which cuts edge $I \leq 4$ and edge $J \leq 4$. Another example is $\mathbf{T}_{I}^{J}$, describing a triangular element where one edge $I \leq 3$ and one node $J \leq 3$ are cut. The edge and node numbering are evident from Fig. 7 Note that only the vertex nodes of a higher-order element are relevant for the subdivision scheme. 


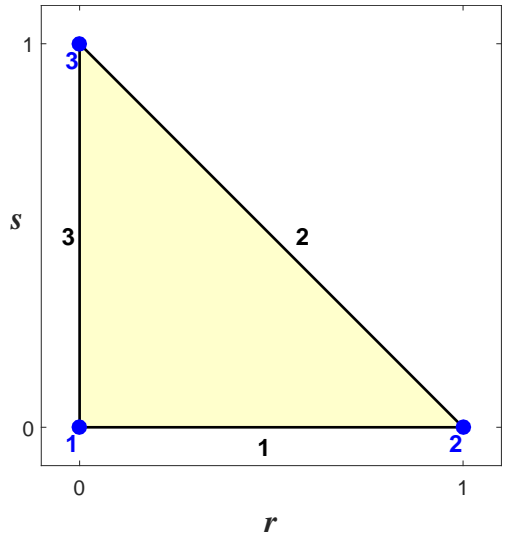

(a) Linear triangular element

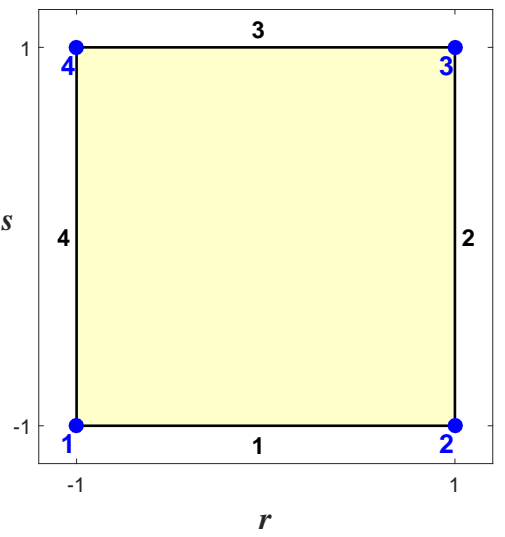

(b) Bi-linear quadrilateral element

Figure 7: Edge and node numbering for (bi-)linear Lagrange elements.

The introduced notation has the advantage that it indicates directly the number of possible topologies. As mentioned in Section 3, a valid cut element has exactly two intersections of the zero level set along the whole element boundary. As a result, all possible cut situations for triangles are described by $\mathbf{T}_{I}^{J}$, $\mathbf{T}_{I J}$ and $\mathbf{T}^{I J}$, with $I \leq 3$ and $J \leq 3$. The same is valid for quadrilaterals, $\mathbf{Q}_{I}^{J}, \mathbf{Q}_{I J}$ and $\mathbf{Q}^{I J}$, with $I \leq 4$ and $J \leq 4$. It is worth recalling that a symmetry in the indices holds, e.g. $\mathbf{T}_{I J}=\mathbf{T}_{J I}$ which changes only the orientation of the normal vector but not the topological situation itself. Consequently, the permutations of $I$ and $J$ for subscripts and superscripts cover all possible topological situations. The only invalid situation is one node that is cut twice by the level set. In the proposed notation that would result in $\mathbf{T}^{I I}$ and $\mathbf{Q}^{J J}$ with $I \leq 3$ and $J \leq 4$.

\subsection{Subdivision strategy for triangles}

A configuration is defined as "local" if the element can be subdivided without influencing neighbour elements. For triangular elements there are two local configurations, that affect only the considered element. Another three non-local configurations are present, influencing the subdivision of one neighbour element. The non-local scheme works successively which means that the (non-local) subdivision scheme produces a local configuration. Once this is done, the local subdivision algorithm yields the desired element patches where some element nodes coincide with the reconstructed interface element.

\subsubsection{Local subdivisions}

The subdivisions, which do not affect the neighbour elements or do not need any information from the neighbour elements to produce a unique subdivision, are referred to as local subdivisions. The configuration in Fig. 8 (a) is the first local topology. It consists of a triangular element divided into a triangle and a quadrilateral by two cut edges. The situation in the figure is labelled as $\mathbf{T}_{13}$, but all situations fulfilling $\mathbf{T}_{I J}, I \leq 3, J \leq 3$ and $I \neq J$ are also included in this topological situation. The other standard topology occurring for triangular elements is given in Fig. 8 (b) as $\mathbf{T}_{2}^{1}$, but again the basic topology covers in total the combinations $\left\{\mathbf{T}_{2}^{1}, \mathbf{T}_{3}^{2}, \mathbf{T}_{1}^{3}\right\}$. In this case a vertex and the opposite edge node are cut, see Fig. 8,b). For this topological case, the element patch is easily subdivided into two triangular elements. This case is particularly important when distinguished points occur e.g. a sudden change in the geometry (e.g. corners) or boundary conditions. This is considered in detail in a follow-up paper.

\subsubsection{Non-local subdivisions}

Some topologies are considered that need a specialized treatment as they influence the neighbour elements. These cases occur in connection with a glancing intersection of the level set with one element edge. Often the problem could also be resolved by a refinement of the background mesh. However, from a computational point of view, it is still preferable to proceed as follows. We go through all elements of the mesh and find the intersections of the level set function with the element boundaries. For edges that are cut twice, the adjacent elements are subdivided as in Fig.9. 9 ). 


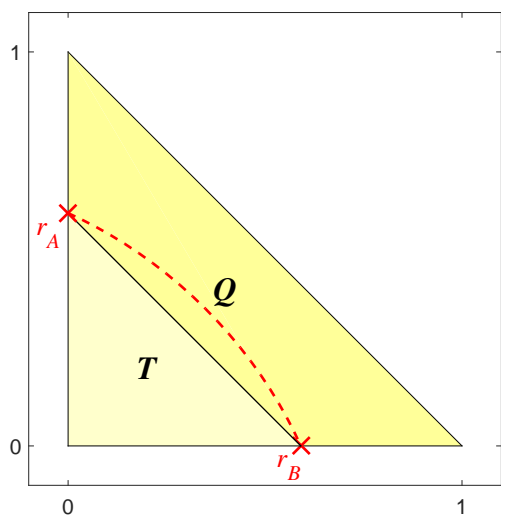

(a) $\mathbf{T}_{13}$

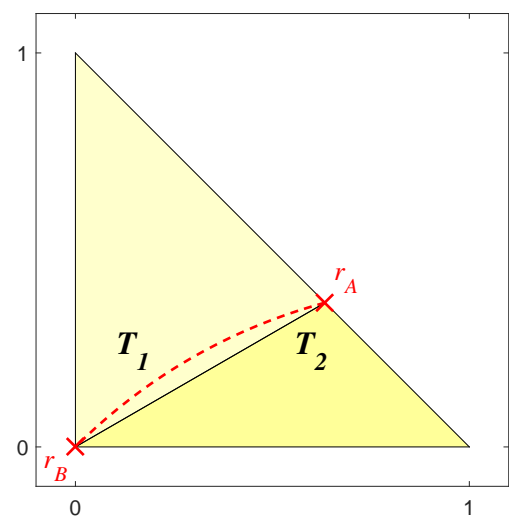

(b) $\mathbf{T}_{2}^{1}$

Figure 8: Local subdivisions for triangles including the zero level set $\phi^{h}$ (a) when two different edges are cut and (b) a node and the opposite edge are cut.

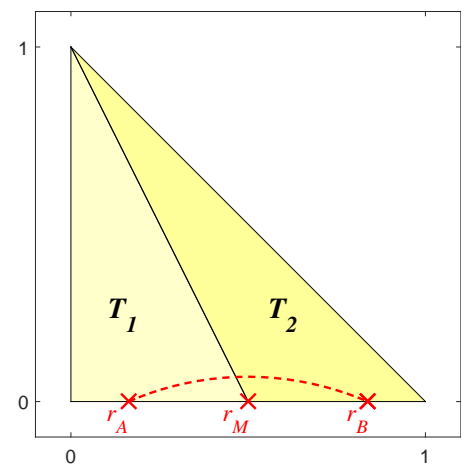

(a) $\mathbf{T}_{11}$

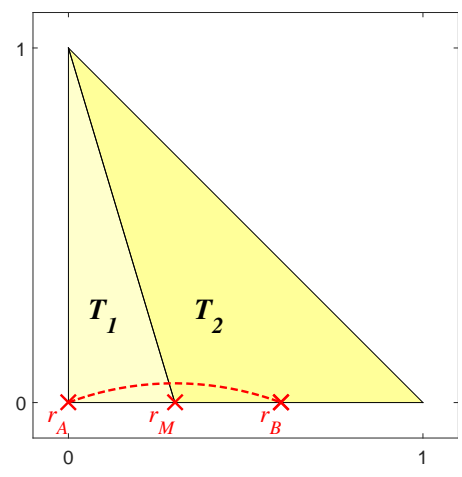

(b) $\mathbf{T}_{1}^{1}$

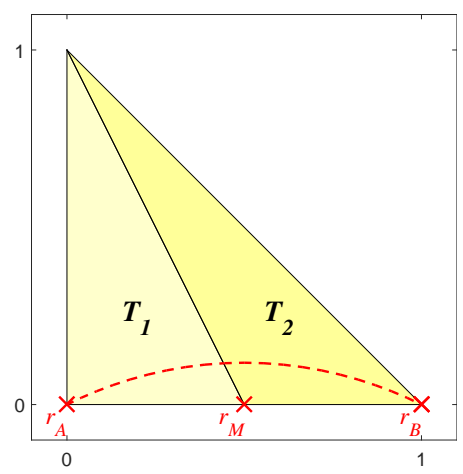

(c) $\mathbf{T}^{12}$

Figure 9: Non-local subdivisions of a triangle as a consequence of (a) one edge cut twice, (b) one cut node with a neighbouring cut edge and (c) two cut nodes.

The element is subdivided in the middle of the interval defined by the two roots on the edge, $\boldsymbol{r}_{M}=\frac{1}{2}\left(\boldsymbol{r}_{A}+\boldsymbol{r}_{B}\right)$. Collectively, the topological combinations $\left\{\mathbf{T}_{11}, \mathbf{T}_{22}, \mathbf{T}_{33}\right\}$ are covered by this subdivision.

The two other cases are depicted in Fig. 9 (b) where a node is cut together with a neighbouring edge, described by $\left\{\mathbf{T}_{1}^{1}, \mathbf{T}_{1}^{2}, \mathbf{T}_{2}^{2}, \mathbf{T}_{2}^{3}, \mathbf{T}_{3}^{3}, \mathbf{T}_{3}^{1}\right\}$, and Fig. 9 (c) where two nodes are cut, described by $\left\{\mathbf{T}^{11}, \mathbf{T}^{22}, \mathbf{T}^{33}\right\}$. The subdivision strategy is similar as before: Both cases can be subdivided into two triangles, with the point $\boldsymbol{r}_{M}$ located in the middle of the intersections of the zero level sets with the element boundary.

Once the non-local subdivisions are executed, all topological cases can be treated by the local subdivision strategy described before.

\subsection{Subdivision strategy for quadrilaterals}

Starting with a mesh composed by quadrilateral elements, one approach would be to convert the cut elements into triangles and apply the scheme for triangles from above. However, this would obviously lead to (slightly) different zero level sets compared to those implied by the interpolation functions in a quadrilateral element. Therefore, we rather develop schemes that work without prior conversion. A total of four local subdivisions are introduced, depending on the cut situation. As before, it is also necessary to consider cases where the neighbour elements are influenced, which are again named non-local subdivisions. 


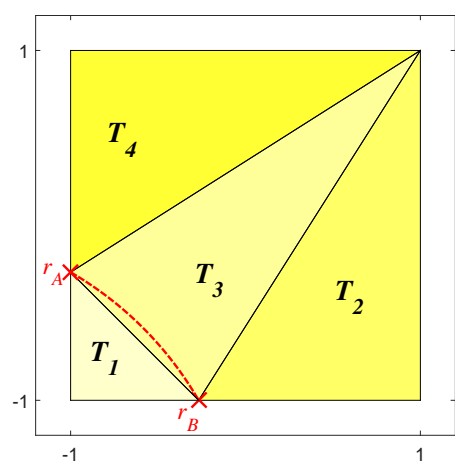

(a) $\mathbf{Q}_{14}$

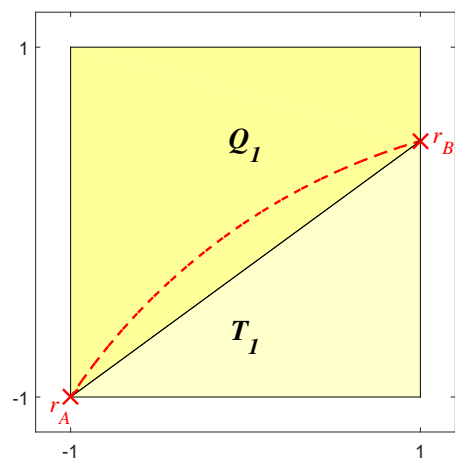

(c) $\mathbf{Q}_{2}^{1}$

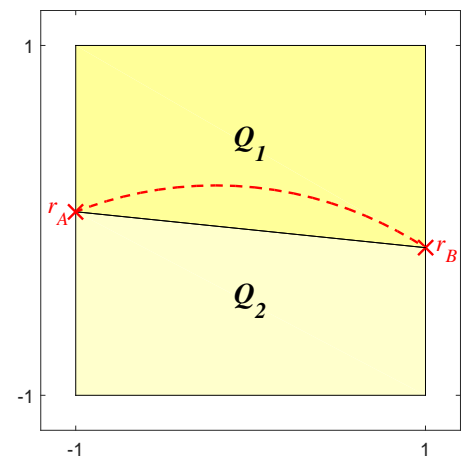

(b) $\mathbf{Q}_{24}$

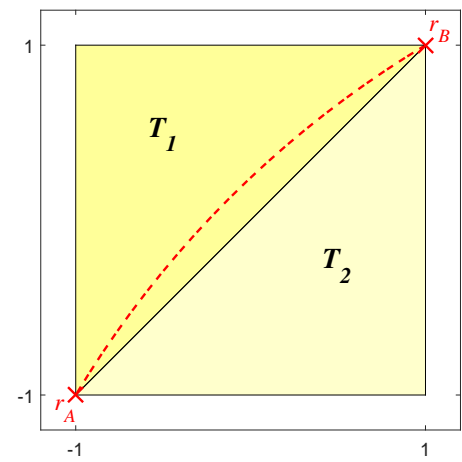

(d) $\mathbf{Q}^{13}$

Figure 10: All variants of local subdivision schemes for a quadrilateral elements: (a) features two neighbouring cut edges, (b) two opposite cute edges, (c) a cut vertex and a non-neighbour cut edge, and (d) two cut opposite vertices.

\subsubsection{Local subdivisions}

All situations featuring two cut adjacent edges are topologically equivalent, see Fig. 10 a) for $\mathbf{Q}_{14}$. The subdivision for this topological case produces four triangular elements. All other topological cases $\left\{\mathbf{Q}_{12}, \mathbf{Q}_{23}, \mathbf{Q}_{34}\right\}$ are also covered with the proposed subdivision. Another case is when two neighbouring edges are cut as depicted in Fig. 10 (b) for $\mathbf{Q}_{24}$. This subdivision creates two quadrilaterals. Again, also the rotated situation $\mathbf{Q}_{13}$, is covered by the subdivision. The first topology considering also nodal cuts is depicted in Fig. 10 (c). In that case, a node and a non-adjacent edge are cut and subdivide the original quadrilateral into a quadrilateral and a triangle. The covered situations are $\left\{\mathbf{Q}_{2}^{1}, \mathbf{Q}_{3}^{1}, \mathbf{Q}_{3}^{2}, \mathbf{Q}_{4}^{2}, \mathbf{Q}_{4}^{3}, \mathbf{Q}_{1}^{3}, \mathbf{Q}_{1}^{4}, \mathbf{Q}_{2}^{4}\right\}$. The last local subdivision is depicted in Fig. 10.(c). This topology features two cut opposite nodes, $\left\{\mathbf{Q}^{13}, \mathbf{Q}^{24}\right\}$, leading to two triangles.

\subsubsection{Non-local subdivisions}

To deal with glancing intersections, as depicted in Fig. 11 a), it is necessary to subdivide the elements adjacent to the considered edge. In total, as for the triangular elements, three different topological cases arise. All situations have in common that one edge is cut twice. The first case, see Fig. 11 a), includes the topologies $\left\{\mathbf{Q}_{11}, \mathbf{Q}_{22}, \mathbf{Q}_{33}\right\}$. The subdivision of this topological case is done by three triangles, whereby the triangles share the point $\boldsymbol{r}_{M}=\frac{1}{2}\left(\boldsymbol{r}_{A}+\boldsymbol{r}_{B}\right)$. All other topological cases are similar to the former one when placing one root, as in Fig. 11. b), or both roots on the vertex nodes, see Fig. 11 (c). The covered situations are then $\left\{\mathbf{Q}_{1}^{1}, \mathbf{Q}_{1}^{2}, \mathbf{Q}_{2}^{2}, \mathbf{Q}_{2}^{3}, \mathbf{Q}_{3}^{3}, \mathbf{Q}_{3}^{4}, \mathbf{Q}_{4}^{4}, \mathbf{Q}_{4}^{1}\right\}$ for one cut vertex and $\left\{\mathbf{Q}^{11}, \mathbf{Q}^{22}, \mathbf{Q}^{33}\right\}$ for two cut vertices.

\subsection{Coordinate mappings}

The task is now to construct a map for all points from a higher-order reference element to the previously defined low-order sub-cells with potentially one higher-order side. The mapping is constructed so that the following properties 


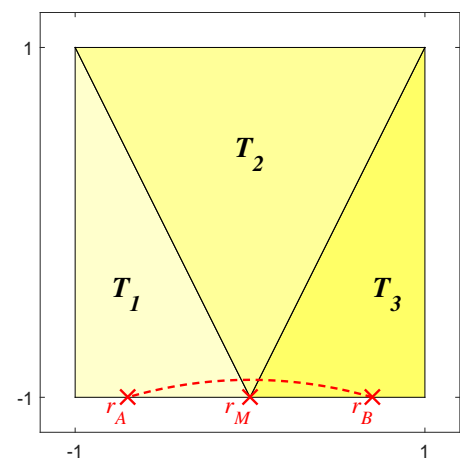

(a) $\mathbf{Q}_{11}$

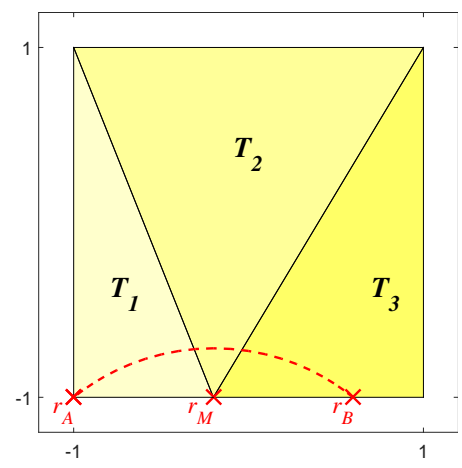

(b) $\mathbf{Q}_{1}^{1}$

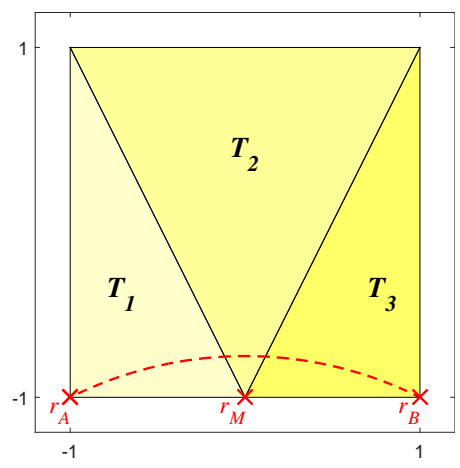

(c) $\mathrm{Q}^{12}$

Figure 11: Non-local subdivisions of a quadrilateral with (a) one edge cut twice, (b) a cut node next to a cut edge, (c) two cut neighbouring nodes.

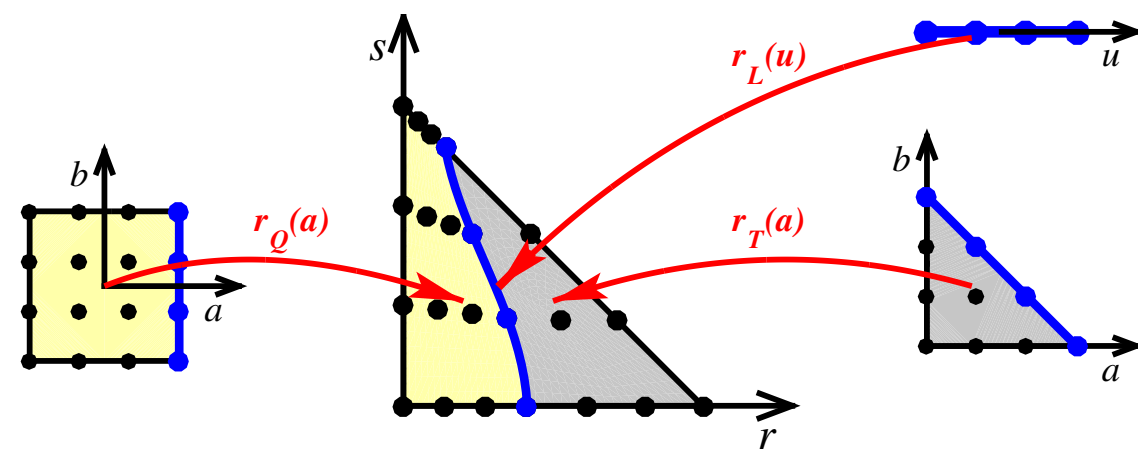

reference background element

with mapped interface and sub-elements

Figure 12: A higher-order background element is cut by the reconstructed interface element $\Gamma_{r}^{h}$ and subdivided into low-order sub-cells defining topology $\mathbf{T}_{12}$. The mapping from the reconstructed element to the coordinates $\boldsymbol{r}$ is given by $\boldsymbol{r}_{L}(u)$. A set of higher-order reference elements in the coordinate system $\boldsymbol{a}$ is introduced. The coordinate transformations $\boldsymbol{r}_{T}(\boldsymbol{a})$ and $\boldsymbol{r}_{Q}(\boldsymbol{a})$ map the higher-order reference sub-elements to the coordinates $\boldsymbol{r}$ such that one special side (in our case always edge 2) matches exactly $\Gamma_{r}^{h}$.

are fulfilled: (i) the element resulting from the coordinate transformation yields optimal interpolation properties and (ii) one element edge aligns with the (curved) higher-order interface element $\Gamma_{r}^{h}$.

As seen from Fig. 12, several element types and corresponding coordinate systems are involved: Central to the whole procedure are the low-order sub-cells (with one higher-order side) described in the coordinate system $r$, as defined above. They define the outer boundary of the higher-order elements resulting from the automatic conformal decomposition. Nevertheless, the mapping for inner points in the sub-cells is not unique, so that inner nodes of the resulting higher-order elements may be placed differently which has important implications on the convergence properties. The higher-order interface element representing the discretized zero level set $\phi^{h}$ is initially defined in the reference coordinate system $u$ and is mapped to the reference element $\Omega_{\boldsymbol{r}}$ by $\boldsymbol{r}(u)$. Finally, there are higher-order reference sub-elements defined in the coordinate system $\boldsymbol{a}$. These sub-elements are used to define the initial location of the nodes and the mapping to $\Omega_{\boldsymbol{r}}$ is denoted as $\boldsymbol{r}(\boldsymbol{a})$.

It is useful to introduce the following definitions related to nodal sets and corresponding shape functions of the higher-order sub-elements in the reference coordinate system $\boldsymbol{a}$. The set of the corner nodes for each element is defined by $I_{\mathrm{lin}}^{\Omega}$. These are three nodes for a triangular and four for a quadrilateral element. From these nodes, either one node (see Fig. 10 (a) topology $\mathbf{Q}_{14}$, elements $\mathbf{T}_{2}$ and $\mathbf{T}_{4}$ ) or two nodes (see Fig. 10 (a) for topology $\mathbf{Q}_{14}$, elements $\mathbf{T}_{1}$ and $\mathbf{T}_{3}$ ) belonging to $I_{\text {side }}^{\Omega} \subset I_{\text {lin }}^{\Omega}$ are on the linear reconstruction when mapped to $\boldsymbol{r}$. For simplicity, we choose a node numbering in the sub-elements such that edge 2 is the one that after the mapping to $r$ will coincide exactly 
with the interface element. The set of nodes on edge 2 is called $I_{\mathrm{ho}}^{\Gamma}$, and the corner nodes are $I_{\text {lin }}^{\Gamma} \subset I_{\text {ho }}^{\Gamma}$. Related to these nodes are the nodal coordinates $\boldsymbol{a}_{i}$ before the mapping and $\boldsymbol{r}_{i}$ after the mapping to the cut reference background element. Note that the nodes in $I_{\text {side }}^{\Omega}$ and $I_{\text {lin }}^{\Gamma}$ superpose in coordinate systems $\boldsymbol{a}$ and $\boldsymbol{r}$. We associate the following set of shape functions with the above-mentioned nodes: $N_{\operatorname{lin}, i}^{\Omega}(\boldsymbol{a})$ for $i \in I_{\operatorname{lin}}^{\Omega}$ are the low-order shape functions of the reference sub-element. $N_{\mathrm{ho}, i}^{\Gamma}(u)$ for $i \in I_{\mathrm{ho}}^{\Gamma}$ are the higher-order shape functions on the special side of the subelement. $N_{\operatorname{lin}, i}^{\Gamma}(u)$ for $i \in I_{\text {lin }}^{\Gamma}$ are low-order shape functions on the special side which coincide with the trace of the low-order shape functions $N_{\text {lin }, i}^{\Omega}(\boldsymbol{a})$ for $i \in I_{\text {side }}^{\Omega}$ on edge 2 .

The aim is to construct a map for all nodal points from $\boldsymbol{a}$ to $\boldsymbol{r}$ based on the known parametrization of the edges (note that $\Gamma_{r}^{h}$ is included in some 2D-elements as a curved edge). The mapping transforms the low-order sub-cells into higher-order elements with one curved edge. Therefore, we adapt a transfinite interpolation technique as described e.g. in [25, 26, 54, 50]. The scheme yields the blending function method for the quadrilaterals [25, 26] and a slightly modified technique for the triangular elements. We start with the mapping for the elements having one node in $I_{\text {side }}^{\Omega}$ (or alternatively the assumption that $\Gamma_{r}^{h}$ is straight). In this case the mapping from $\boldsymbol{a}$ to $\boldsymbol{r}$ is defined completely by the vertices and is given by the standard isoparametric mapping for bi-linear elements as

$$
\boldsymbol{r}_{m}\left(\boldsymbol{a}_{n}\right)=\sum_{i \in I_{\operatorname{lin}}^{\Omega}} N_{\operatorname{lin}, i}^{\Omega}\left(\boldsymbol{a}_{n}\right) \cdot \boldsymbol{r}_{\operatorname{lin}, i}^{\Omega}, \quad \boldsymbol{a}_{n}, \boldsymbol{r}_{m} \in \mathbb{R}^{2} .
$$

The coordinates $\boldsymbol{a}_{n}$ and $\boldsymbol{r}_{m}$ are the nodal coordinates and $\boldsymbol{r}_{\text {lin, } i}^{\Omega}$ are the corner nodes of a sub-element in the cut reference background element in the corresponding coordinate systems. To include the more elaborate case of a curved higher-order interface element, an additional term to the standard isoparametric mapping must be included, defined as

$$
\boldsymbol{r}_{m}\left(\boldsymbol{a}_{n}\right)=\sum_{i \in I_{\operatorname{lin}}^{\Omega}} N_{\operatorname{lin}, i}^{\Omega}\left(\boldsymbol{a}_{n}\right) \cdot \boldsymbol{r}_{\operatorname{lin}, i}^{\Omega}+\boldsymbol{M}\left(\boldsymbol{a}_{n}\right), \quad \boldsymbol{a}_{n}, \boldsymbol{r}_{m} \in \mathbb{R}^{2} .
$$

Equations (4) and (5) are valid for any point inside $\Omega_{a}$. Note that Eq. (5) must include Eq. (4) as a special case of an element with only straight edges. Therefore the additional vector function $\boldsymbol{M}(\boldsymbol{a}) \in \mathbb{R}^{d}$ must be defined such that it vanishes for elements with only straight edges. It is interesting to note that the definition of the geometric mapping (5) formally matches enriched approximations such as those used in the XFEM [5, 38, 23, 2, 3].

For the definition of $\boldsymbol{M}(\boldsymbol{a})$, we impose two important constraints:

1. The corner nodes of the higher-order interface element $I_{\text {lin }}^{\Gamma}$ match the corner nodes of the special side of the low-order element $I_{\text {side }}^{\Omega}$ in the coordinate system $r$.

2. Those edges of the low-order elements that are not on the special side shall remain straight after the mapping.

This leads to the requirement that

$$
\boldsymbol{M}\left(\boldsymbol{a}_{i}\right)=\mathbf{0} \quad \forall i \in I_{\mathrm{lin}}^{\Omega} .
$$

We find that this can be achieved when splitting $M(a)$ into the product

$$
\boldsymbol{M}(\boldsymbol{a})=\psi(\boldsymbol{a}) \cdot \boldsymbol{f}(u(\boldsymbol{a}))
$$

with the definition of $\boldsymbol{f}(u(\boldsymbol{a}))$ as

$$
\boldsymbol{f}(u(\boldsymbol{a}))=\sum_{i \in I_{\mathrm{ho}}^{\Gamma}} N_{\mathrm{ho}, i}^{\Gamma}(u(\boldsymbol{a})) \cdot \boldsymbol{r}_{i}^{\Gamma}-\sum_{i \in I_{\mathrm{lin}}^{\Gamma}} N_{\operatorname{lin}, i}^{\Gamma}(u(\boldsymbol{a})) \cdot \boldsymbol{r}_{i}^{\Gamma}
$$

The coordinates $\boldsymbol{r}_{i}^{\Gamma}$ are thereby the element nodes of the reconstructed interface elements in the coordinate system $\boldsymbol{r}$. This function corresponds geometrically to subtracting the linear connection of the nodes in $I_{\operatorname{lin}}^{\Gamma}$ from the curved higher-order side. The function $\psi$ is differently defined for triangular and quadrilateral elements and describes the procedure how the curved edge is blended into an element with only straight edges. However, note that $\psi$ is not unique and there are many conceivable alternatives to it.

As theoretically devised by [15] for 2D simplex elements and extended by [32] and [8] for higher dimensions, certain smoothness properties have to be fulfilled by the desired coordinate transformation. In general, it can be stated that the geometrical mapping that is defined by the shape functions in the reference domain should also be polynomial in the real space. Further explanations and extensions to transfinite mappings are found in [50],[55]. 


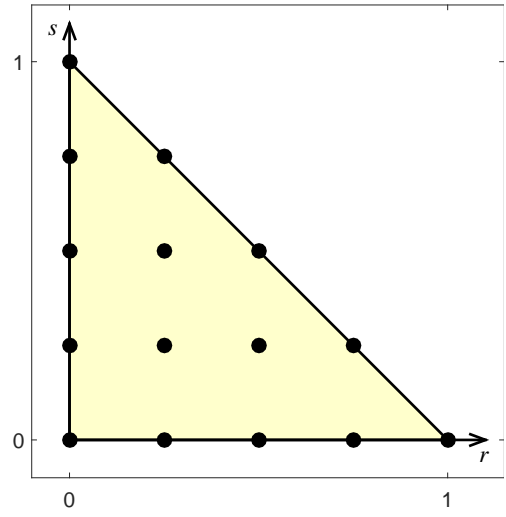

(a)

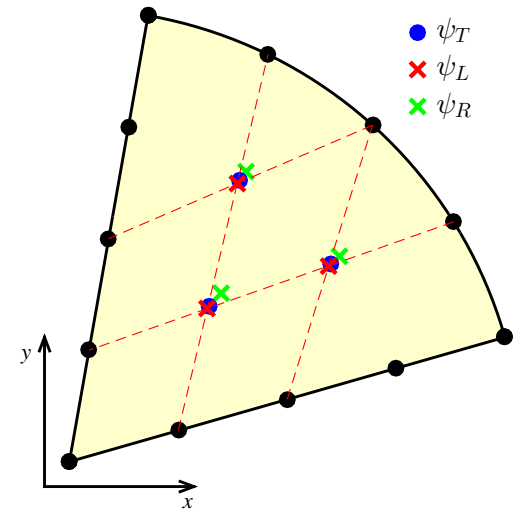

(b)

Figure 13: (a) Node distribution inside a reference triangle for order $m=4$; (b) location of internal nodes for different choices of $\psi$ in case of a circular arc parametrization of edge 2 .

\subsubsection{Mapping for curved triangular elements}

It remains to define $u=u_{T}(\boldsymbol{a})$ and $\psi$. The parametrization of edge 2 is done by means of vertex functions and gives $u_{T}(\boldsymbol{a})=N_{\operatorname{lin}, 3}^{\Omega}(\boldsymbol{a})-N_{\operatorname{lin}, 2}^{\Omega}(\boldsymbol{a})$. The choice of $\psi$ is then given by [50] as

$$
\psi=\psi_{T}:=\frac{\prod_{i \in I_{\operatorname{lin}}^{\Omega}} N_{\operatorname{lin}, i}^{\Omega}(\boldsymbol{a})}{\prod_{i \in I_{\operatorname{lin}}^{\Gamma}} N_{\operatorname{lin}, i}^{\Gamma}\left(u_{T}(\boldsymbol{a})\right)} .
$$

The mapping is valid for all points with exception of nodal points on $I_{\text {side }}^{\Omega}$ where a division by zero arises. For these points, however, the mapping of the vertices is defined by the standard isoparametric mapping. Anyway, also alternative choices of $\psi$ are conceivable, e.g. using the ramp function (which is in fact the mapping of choice for the quadrilaterals), hence $\psi_{R}:=R(\boldsymbol{a})=\sum_{I_{\text {side }}^{\Omega}} N_{\text {lin,i }}^{\Omega}(\boldsymbol{a})$. Another choice is to use mappings such as in [15, 47, 32]. In this case, intersections of the boundary nodes are taken as internal nodes, this is further on designated as $\psi_{L}$ (Lenoirmapping). An issue with this approach is that the extension to $3 \mathrm{D}$ is not trivial. Differences in the mappings are plotted in Fig. 13 In the following chapter, we investigate the influence of the choices of $\psi$ on the convergence rates. Note that in the case of straight edges, the enrichment function $\boldsymbol{M}_{T}(\boldsymbol{a})=\mathbf{0}$, and consequently a standard bi-linear mapping is obtained.

In Fig. 13 the inner node distributions are shown for different versions of $\psi$. The edge is hereby parametrized by equally spaced nodes on a quarter circle. Note that the mapping for the boundary nodes coincides for all choices of $\psi$ but differs inside. As shall be seen below, the node distribution inside the elements is of large importance for the achieved convergence properties.

\subsubsection{Mapping for curved quadrilateral elements}

For quadrilateral elements, the optimal mapping is found using the blending function method. Introduced by [26] for transfinite (in general non-polynomial) mappings, it can also be used for isoparametric edge approximations. In this case, a scalar vertex or ramp function is defined by

$$
\psi:=R(\boldsymbol{a})=\sum_{I_{\text {side }}^{\Omega}} N_{\operatorname{lin}, i}^{\Omega}(\boldsymbol{a}) .
$$

The enrichment function $M(\boldsymbol{a})$ for quadrilaterals is then defined as the linearly blended map

$$
\boldsymbol{M}_{Q}(\boldsymbol{a})=R(\boldsymbol{a}) \cdot \boldsymbol{f}\left(u_{Q}(\boldsymbol{a})\right) .
$$




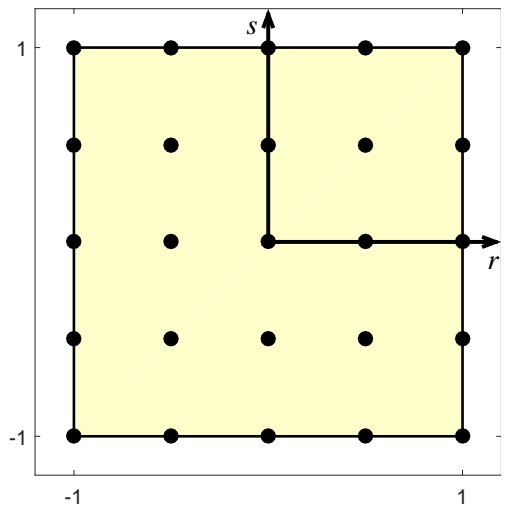

(a)

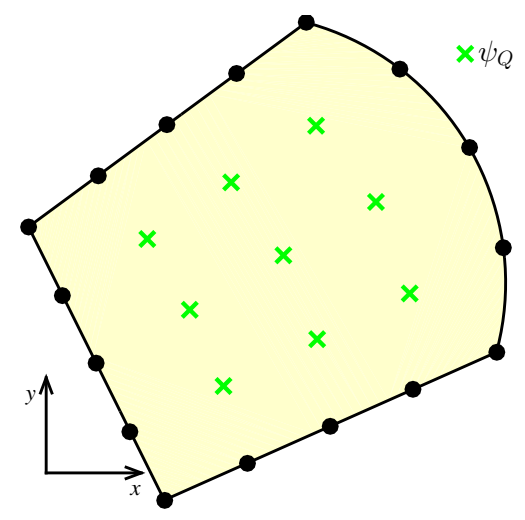

(b)

Figure 14: (a) Node distribution for a quadrilateral element of order $m=4$, and (b) nodal distribution using the blending function method for a circular arc parametrization of edge 2 and equidistant node distribution on the arc.

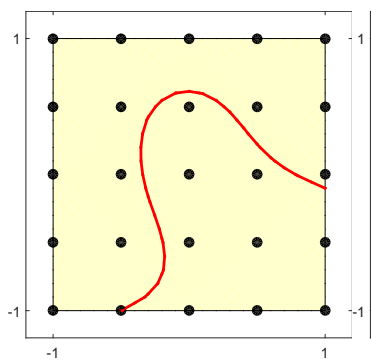

(a)

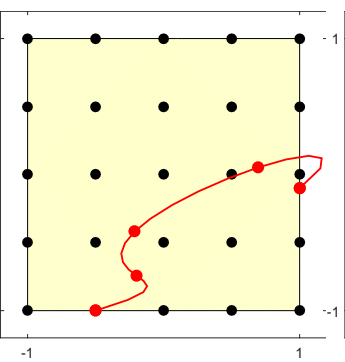

(b)

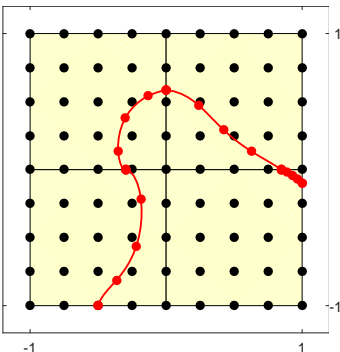

(c)

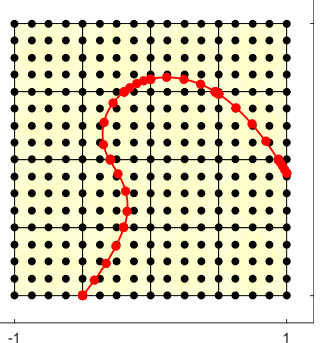

(d)

Figure 15: (a) Exact zero level set inside a higher-order quadrilateral element. (b) The reconstruction fails due to the high curvature of the level set. (c) and (d) The problem is resolved by a uniform refinement of the element until no negative Jacobians are detected.

For quadrilateral elements the parametrization of edge 2 is given by $u_{Q}(\boldsymbol{a})=b$. It is seen that $\boldsymbol{f}\left(u_{Q, i}\right)=\mathbf{0}$ for $i \in I_{\text {lin }}^{\Gamma}$ and, hence, also for the nodes in $I_{\text {side }}^{\Omega}$. Furthermore, $R\left(\boldsymbol{a}_{i}\right)=0$ for $i \in I_{\text {lin }}^{\Omega} \backslash I_{\text {side }}^{\Omega}$, i.e. for all remaining nodes in $I_{\text {lin }}^{\Omega}$. Again, if edge 2 is straight, the enrichment function $M_{Q}(\boldsymbol{a})=\mathbf{0}$, and a standard low-order isoparametric mapping is obtained.

\subsection{Invalid topologies}

As mentioned in Section 3 , the assessment as a valid or invalid topology is based on level set data on the boundary and does not take into account the shape of the level set inside the element. However, in some valid configurations the reconstruction may fail if the curvature of the level set function is too high. A topological situation leading to such a case is shown in Fig. 15. (a). The reconstruction, see Fig. 15.b) fails clearly, also the Runge phenomenon is apparent. However, as the mesh is successively refined, see Fig. 15.(c) and Fig. 15.(d), the situation is finally resolved. For the check, a finite number of points is chosen and the Jacobian is evaluated. Here the points are chosen as the integration points of the elements that emerge from the subdivision scheme but also other choices are possible. The sub-elements resulting from the decomposition are valid if the Jacobian is strictly positive at all discrete points.

\subsection{Influence of the subdivision scheme on the condition number}

An obvious consequence of the proposed methodology is the presence of awkward element shapes: Very small inner angles may occur and the size of neighbouring elements can vary strongly. This may lead to poorly conditioned system matrices, see e.g. [20]. Some remedies for this problem are to use preconditioning schemes or simple mesh manipulation procedures to prevent very small angles. The influence on the condition number is shown here using the 


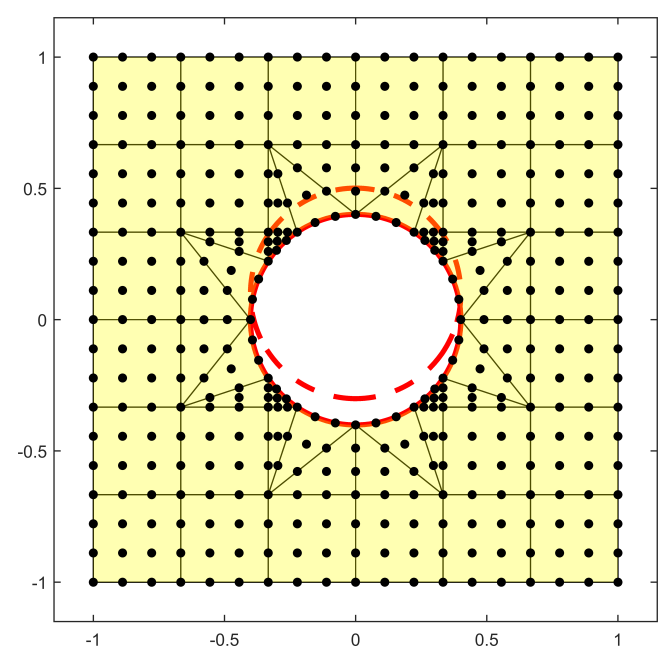

(a)

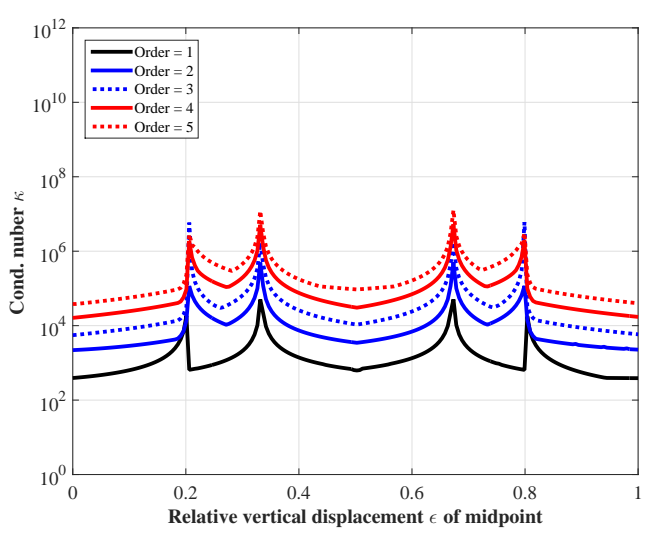

(b)

Figure 16: (a) Moving discontinuity in a structured mesh and (b) condition number in dependence of the relative displacement of the discontinuity.

example of a plate with a hole, which is later investigated in Section 5 . The mesh consists in total of 36 elements, the element order is varied between $m=1$ and $m=5$. The circular inclusion is moved vertically and for each case the condition number is computed and shown in Fig. 16 (b).

Note that the peaks that represent a very high condition number in the system of equations (resulting from a FEanalysis of the plate with hole) emerge only for very few geometries in a small range of the relative displacement. It is not difficult to identify nodes in the background mesh which cause these high condition numbers. Several techniques are available to regulate the situation. Among them are node manipulation schemes as in [34, 44, 29] and stabilization methods as in [34]. Herein, direct solvers are employed for the solution of the system matrices and none of these techniques are employed.

\section{Numerical results}

In order to illustrate the convergence properties and the stability of the proposed method we present four different examples with meshes involving non-fitting interfaces. In the test cases, we focus on (i) the quality of interface reconstructions, (ii) the approximation properties in the context of inner-element boundaries and interfaces, and (iii) the conditioning of the system of equations. The first example is a geometry approximation using a smooth level set function defining an interface. The second example investigates an approximation problem $\left(L_{2}\right.$-projection) of a smooth function on a remeshed, implicitly defined circular domain. And finally, the third and fourth example are boundary value problems in linear elasticity. The third example is a bi-material problem and is representative for methods dealing with (weak) discontinuities as e.g. the XFEM. The fourth example is used to demonstrate the capability of the procedure for a problem typical for fictitious domain methods. Both BVPs are often used as benchmarks for the performance of the $p$-version of the FEM.

\subsection{Interface reconstruction of a flower-shaped inclusion}

Given a reasonably well graded background mesh, this example shows that using the proposed reconstruction scheme fairly complicated domains can be automatically remeshed. The example is inspired by [33, 31] with the analytical level set function (which could e.g. describe the boundary of an inclusion) given by

$$
\phi(\boldsymbol{x}, \theta)=\|\boldsymbol{x}\|-(r+\alpha \sin (\omega \theta))
$$

where $\|\boldsymbol{x}\|$ is the location of a considered point in $\Omega^{h}, \theta \in[0,2 \pi]$ the angle connecting the origin and $\boldsymbol{x}$, and $\{r, \alpha, \omega\}$ a set of variables determining the shape of the inclusion. For the considered example these variables are chosen as 


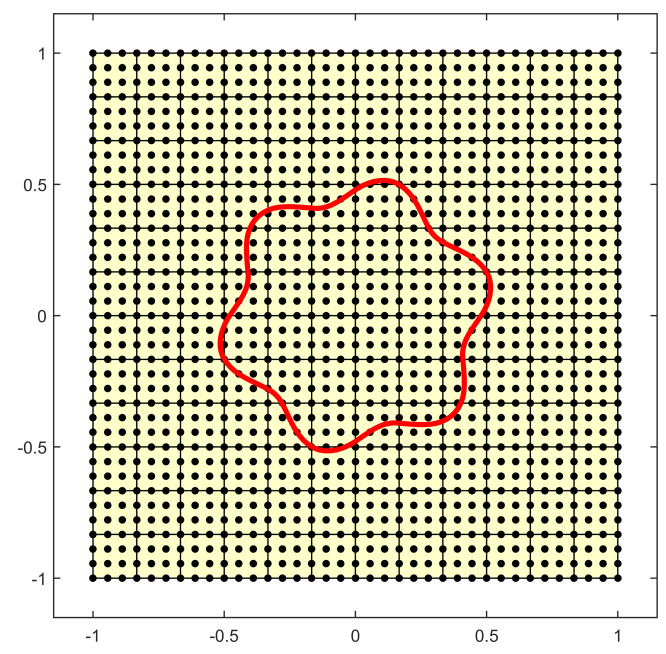

(a) Cartesian background mesh

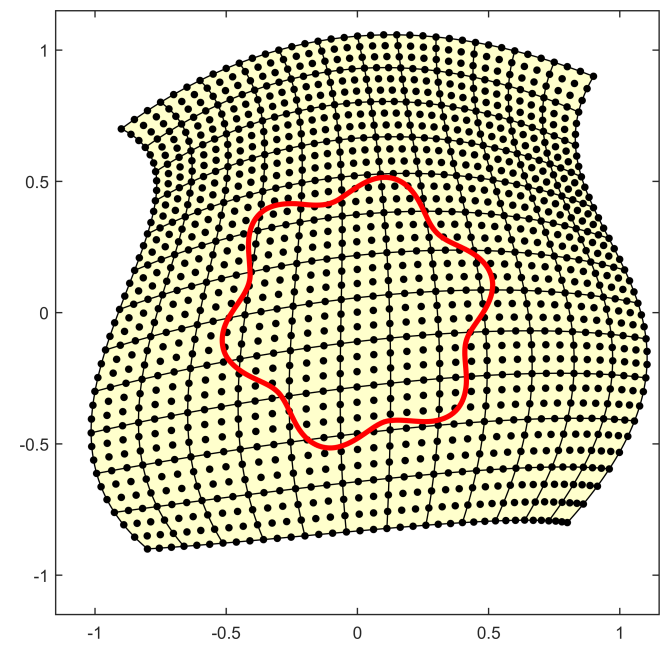

(b) Deformed background mesh

Figure 17: Higher-order background meshes with a flower-like interface.

$\{r=0.48, \alpha=0.05, \omega=6\}$. In Fig. 17(a), a structured Cartesian background mesh is shown and in Fig. 17.b) a deformed background mesh. In the figures, quadrilateral elements of order $m=3$ are shown. Also the interface is seen, which is the zero level set of Eq. (10).

We study the error at integration points which are distributed inside the reconstructed interface elements and evaluate the analytical level set function in these points. Because the interface elements provide an approximation of the zero level set, a suitable error measure is defined as

$$
\varepsilon=\sqrt{\int_{\Gamma} \phi\left(\boldsymbol{x}_{\Gamma}\right)^{2} \mathrm{~d} \Gamma} .
$$

There, $\boldsymbol{x}_{\Gamma}$ are integration points in the reconstructed interface and $\phi\left(\boldsymbol{x}_{\Gamma}\right)$ is the analytical level set function evaluated at $\boldsymbol{x}_{\Gamma}$. The level set function is evaluated at the nodes of the background mesh and interpolated inside the domain using finite element functions. Note that the Newton-Raphson procedure from Section 3 ensures that the element nodes are "exactly" on the zero level set of $\phi^{h}(\boldsymbol{x})$ (with a tolerance of $\approx 10^{-12}$ ). However, for the implied interface element in-between, and consequently also the integration points, a slight deviation from the zero level set of $\phi^{h}$ occurs. The error norm in Eq. 11) accounts for the error in $\phi^{h}$ compared to $\phi$ and for the error in the interface elements in approximating the zero level set of $\phi^{h}$.

In Fig. 18, the automatically generated conformal meshes are visualized. It is emphasized once more, that in general a mixed mesh consisting of triangular and quadrilateral elements results. For clarity the element nodes are not plotted in the meshes.

The analysis is carried out using a series of embedded meshes, indicated by the characteristic element length $h=\operatorname{diam}(K)$, measured in the background mesh (before the decomposition). As shown in Fig. 19.(a) and Fig. 19.b), the convergence properties of the method, based on the Cartesian background mesh as well as on the deformed background mesh, are optimal after the automatic remeshing. Note that no node moving was applied and some of the elements look quite awkward. Nevertheless all elements resulting from the subdivision procedure are topologically valid. However, for higher curvatures inside the elements it can be advisable to apply some mesh manipulations before the subdivision scheme is applied.

\subsection{Approximation problem with circular inclusion}

The second example shows that the automatic decomposition of the background mesh retains optimal approximation properties of the standard $L_{2}$-projection. Also the convergence properties of the different mappings for 


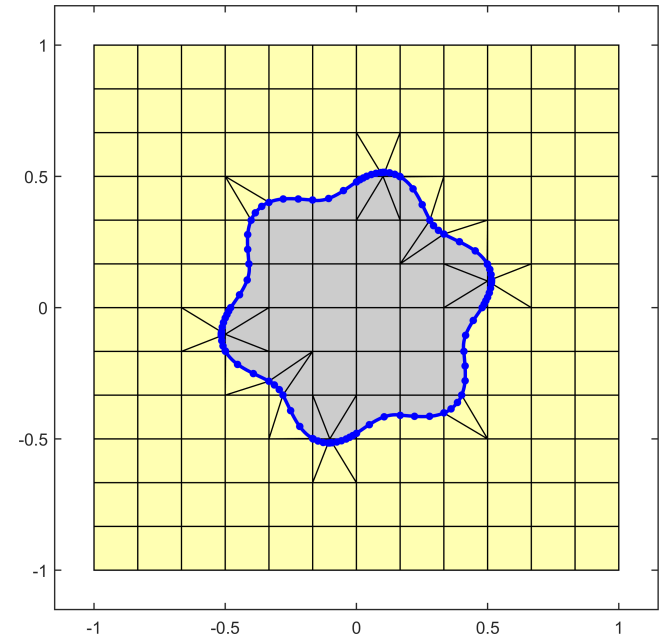

(a) Cartesian background mesh

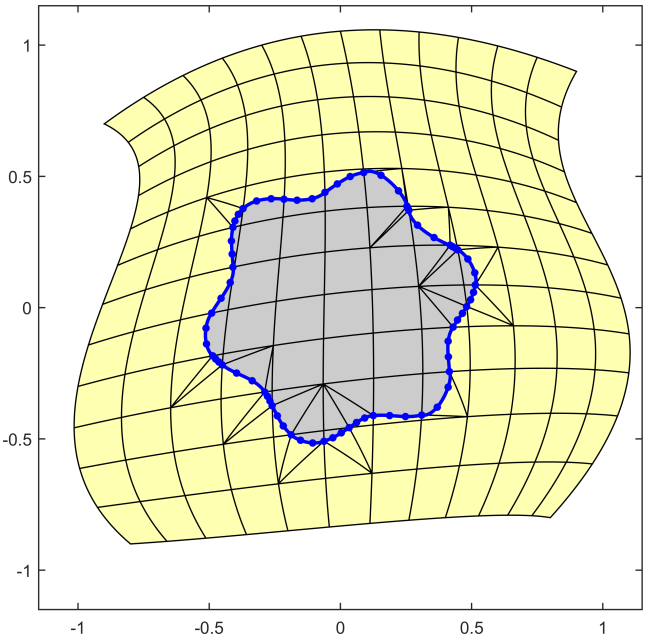

(b) Deformed background mesh

Figure 18: Updated meshes aligning with the inclusion for (a) a Cartesian background mesh and (b) a deformed background mesh.

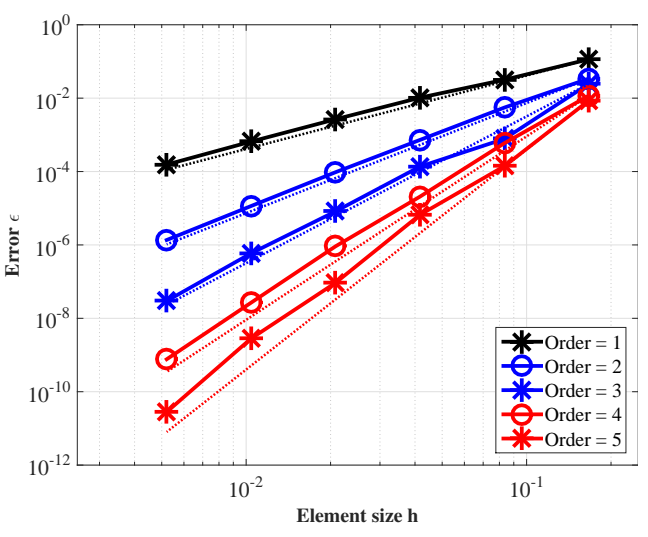

(a) Cartesian background mesh

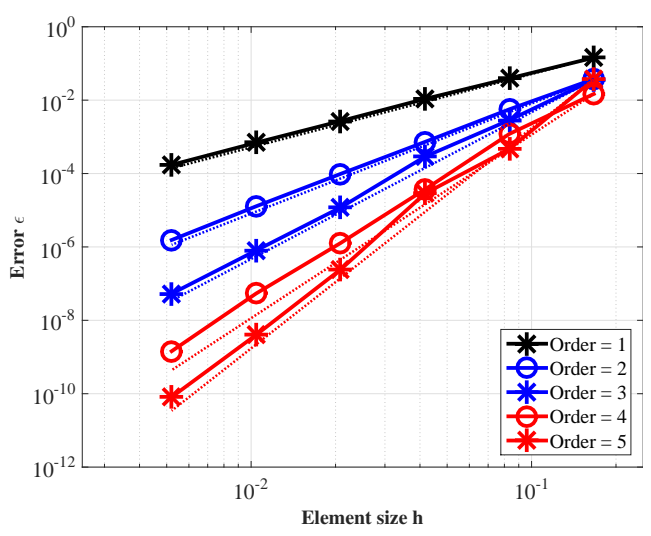

(b) Deformed background mesh

Figure 19: $L_{2}$-error, as defined in Eq. [11, of the interface reconstruction. 


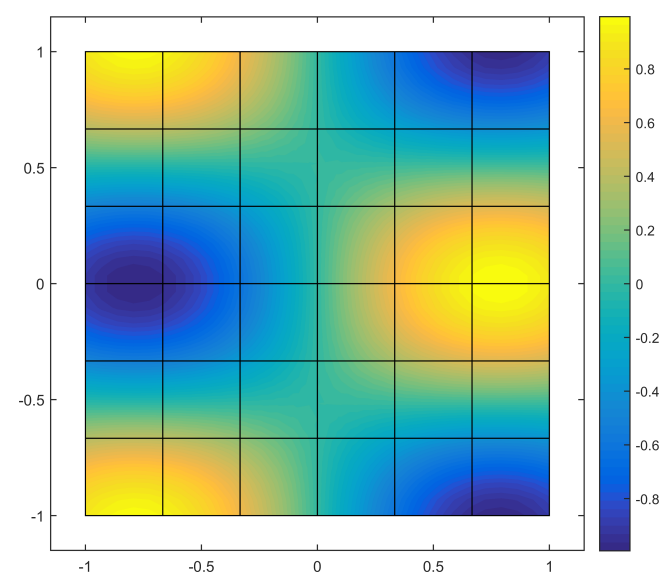

(a) Cartesian background mesh

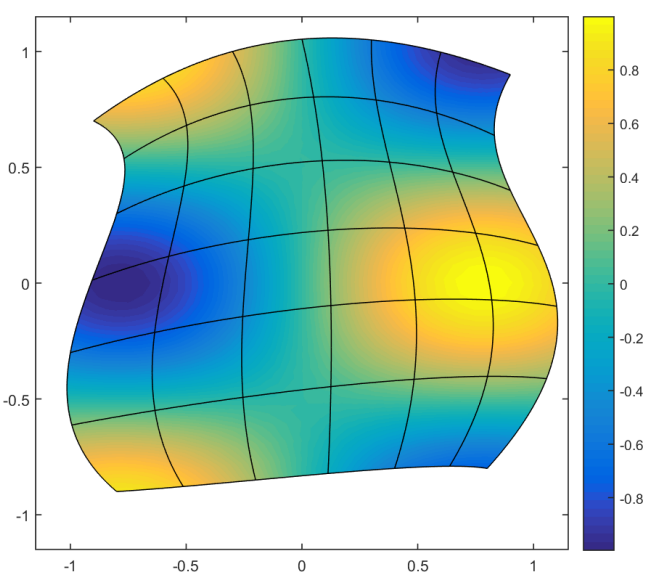

(b) Deformed background mesh

Figure 20: Plot of the function for which an $L_{2}$-projection is made.

triangular elements, as introduced in Section 4, are investigated. Again, a Cartesian and a deformed background mesh, respectively, are automatically decomposed to align with a circular inclusion, see Fig. 21.a) and Fig. 21. (b). The radius of the inclusion is given as $r=0.4$ and centred in the origin. The function to be interpolated is given as $f(x, y)=\sin (2 x) \cos (3 y)$ and therefore smooth over the whole domain. In Fig. 20.a) and Fig. 20.b) plots of the function over the background meshes are shown.

The error is measured in the standard $L_{2}$-norm as well as in the $H_{E}$-norm, to show that in both error measures optimal convergence rates are obtained.

To begin with, we consider the approximation error of $f$ in the $L_{2}$-norm defined by

$$
\varepsilon_{L_{2}}=\sqrt{\int_{\Omega} \frac{\left(f(\boldsymbol{x})-f^{h}(\boldsymbol{x})\right)^{2}}{f(\boldsymbol{x})^{2}} \mathrm{~d} \Omega} \quad \forall \boldsymbol{x} \in \Omega
$$

As depicted in Fig. 22, optimal convergence rates are achieved. Also the gradient approximation error is investigated in the same way, the error is measured in the energy norm $H_{E}$

$$
\varepsilon_{H_{E}}=\sqrt{\int_{\Omega} \frac{\left(\nabla f(\boldsymbol{x})-\nabla f^{h}(\boldsymbol{x})\right)^{2}}{\nabla f^{h}(\boldsymbol{x})^{2}}} \mathrm{~d} \Omega \quad \forall \boldsymbol{x} \in \Omega
$$

The results in Fig. 22 and Fig. 23 show that the remeshing strategy, indeed, achieves optimal convergence properties asymptotically, in both the $L_{2}$-norm and the $H_{E}$-norm.

For the sake of completeness, the $L_{2}$-error for the other mappings of the element nodes to the sub-cells, discussed in Section 4, namely the Lenoir-mapping and the blending function mapping, is also shown for the Cartesian background mesh. The Lenoir-mapping gives optimal approximation properties (but is more difficult to implement than the actually applied mapping). The blending function mapping, on the contrary, clearly gives sub-optimal results and degrades the convergence rates significantly. This is due to the properties of the Jacobian and well-known in the meshing community.

\subsection{Bi-material boundary value problem}

As a third example, a plate with a circular inclusion is considered to illustrate the performance for the approximation of general boundary-value problems with a curved (weak) discontinuity. The example is a well known benchmark problem for higher-order extended finite element procedures, see e.g. [13, 52]. The error in the displacements (measured in the $L_{2}$-norm), the error in stresses (measured in the $H_{E}$-norm) and also the condition number of the resulting system of equations are investigated. Regarding the employed error measures for the convergence studies for this and 


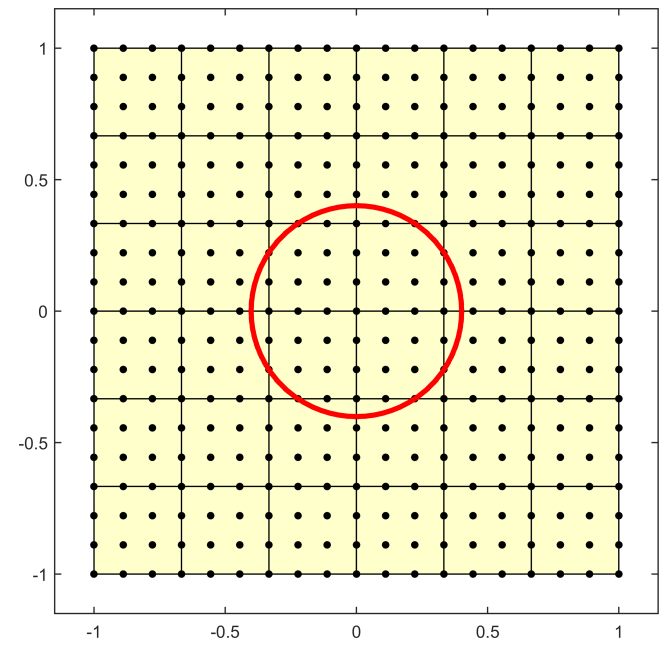

(a) Cartesian background mesh

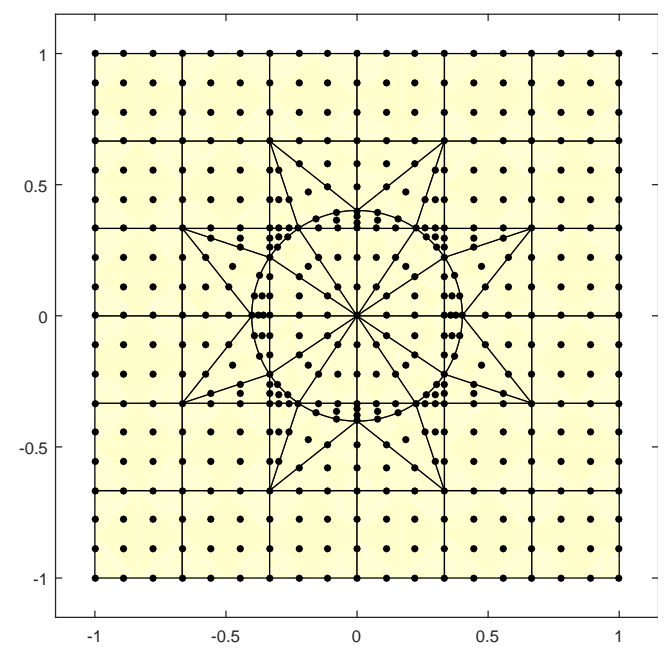

(c) Conformal mesh based on (a)

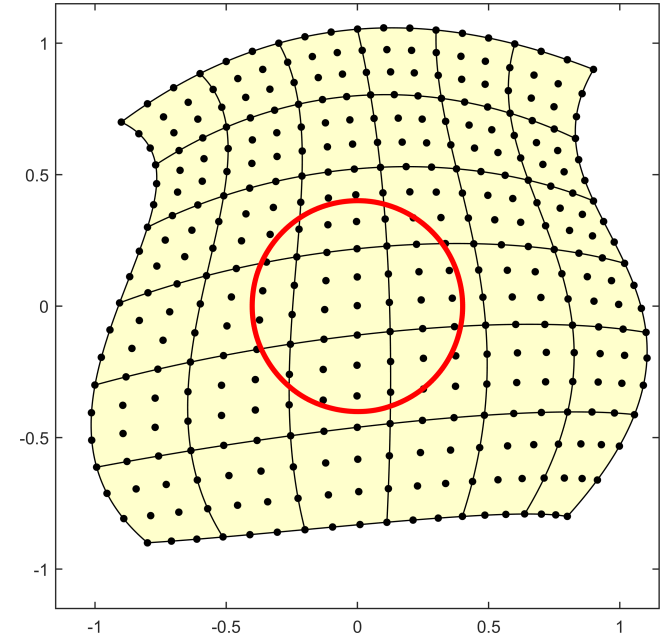

(b) Deformed background mesh

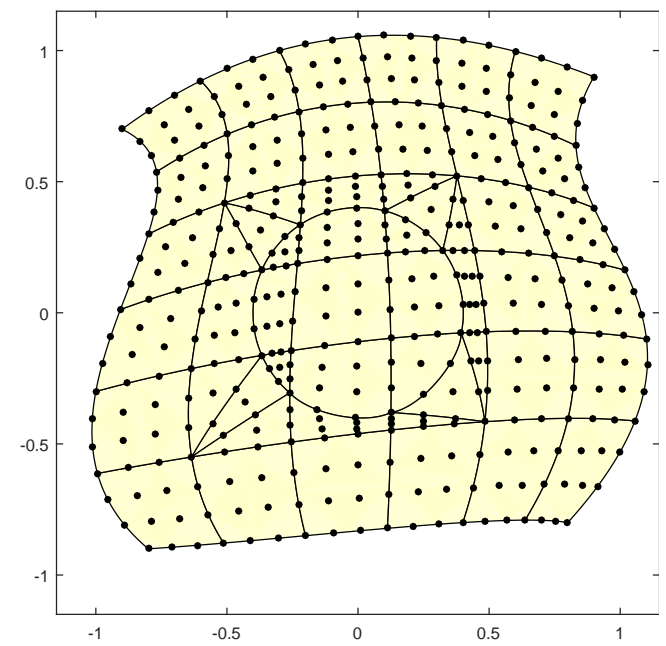

(d) Conformal mesh based on (b)

Figure 21: Plot of the involved meshes: (a) and (b) are background meshes, (c) and (d) show the automatically generated conforming meshes. 


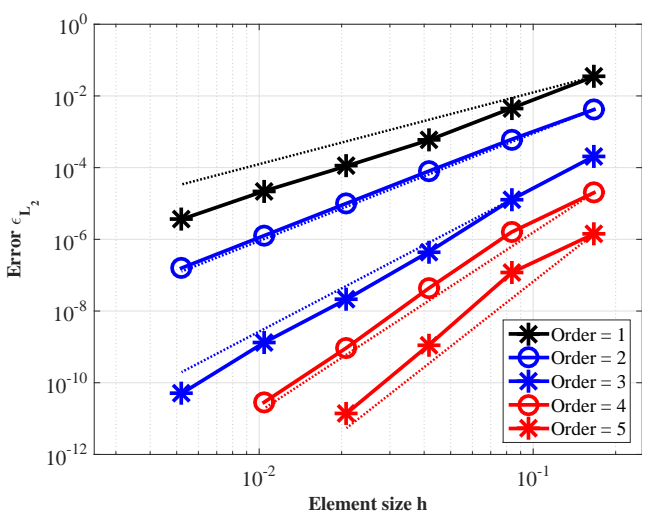

(a) Cartesian background mesh

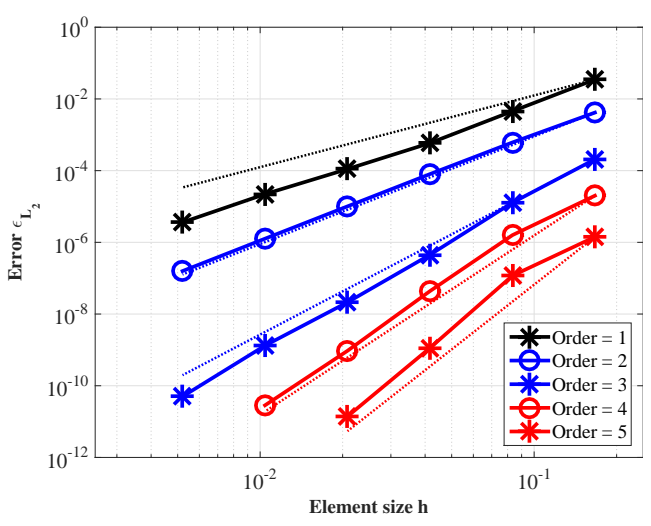

(b) Deformed background mesh

Figure 22: Approximation error measured in the $L_{2}$-norm.

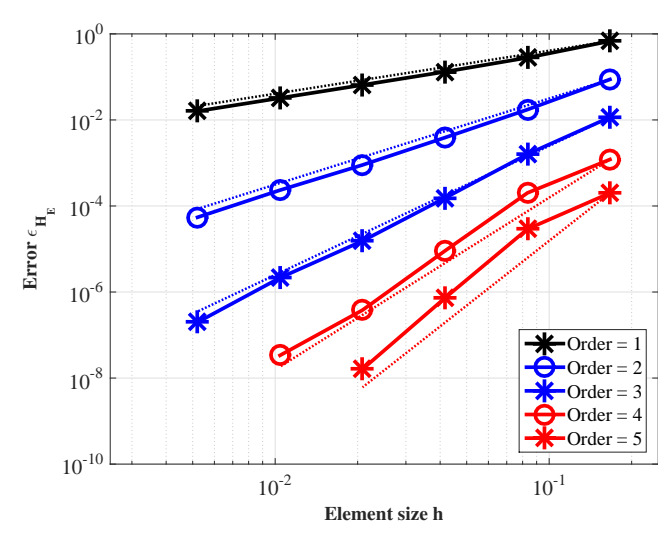

(a) Cartesian background mesh

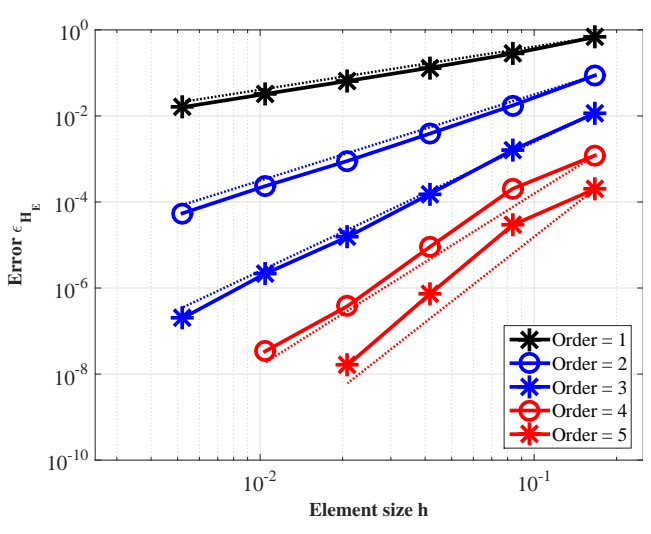

(b) Deformed background mesh

Figure 23: Approximation error measured in the $H_{E}$-Norm

also for the next example, using the analytical solution $\boldsymbol{u}$ and the finite element solution $\boldsymbol{u}^{h}$, the $L_{2}$-norm on $\Omega$ is defined as

$$
\varepsilon_{L_{2}}=\left\|\boldsymbol{u}-\boldsymbol{u}^{h}\right\|_{L_{2}(\Omega)}=\sqrt{\int\left(\boldsymbol{u}-\boldsymbol{u}^{h}\right)^{\top}\left(\boldsymbol{u}-\boldsymbol{u}^{h}\right) \mathrm{d} \Omega} .
$$

To investigate the convergence behaviour of the function derivatives, we use the strain energy $H_{E}$, defined as

$$
\varepsilon_{H_{E}}=\left\|\boldsymbol{u}-\boldsymbol{u}^{h}\right\|_{H_{E}(\Omega)}=\sqrt{\int\left(\boldsymbol{\epsilon}-\boldsymbol{\epsilon}^{h}\right)^{\top} \mathbf{C}\left(\boldsymbol{\epsilon}-\boldsymbol{\epsilon}^{h}\right) \mathrm{d} \Omega}
$$

with $\mathbf{C}$ being the elastic stiffness tensor and $\boldsymbol{\epsilon}(\boldsymbol{u})$ and $\boldsymbol{\epsilon}^{h}\left(\boldsymbol{u}^{h}\right)$ the strain fields of the exact solution and the finite element approximation, respectively. We use relative versions of these two norms, therefore $\varepsilon_{L_{2}(\Omega)}=\| \boldsymbol{u}-$ $\boldsymbol{u}^{h}\left\|_{L_{2}(\Omega)} /\right\| \boldsymbol{u} \|_{L_{2}(\Omega)}$ and $\varepsilon_{H_{E}(\Omega)}=\left\|\boldsymbol{u}-\boldsymbol{u}^{h}\right\|_{H_{E}(\Omega)} /\|\boldsymbol{u}\|_{H_{E}(\Omega)}$. Assuming that a $C_{\infty}$-smooth function is approximated on a regular domain using a polynomial of degree $k$ and with a discretization aligning with the interface, optimal convergence rates are expected, see [53, 51]. Subject to these limitations, the convergence rates in the error norms defined above are

$$
\left\|\boldsymbol{u}-\boldsymbol{u}^{h}\right\|_{L_{2}(\Omega)}=\mathcal{O}\left(h^{k+1}\right) \quad \text { and } \quad\left\|\boldsymbol{u}-\boldsymbol{u}^{h}\right\|_{H_{E}(\Omega)}=\mathcal{O}\left(h^{k}\right) .
$$




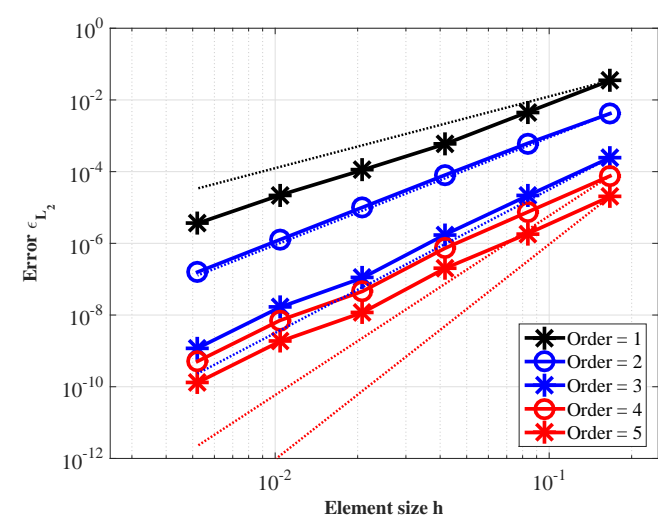

(a) Blending function mapping

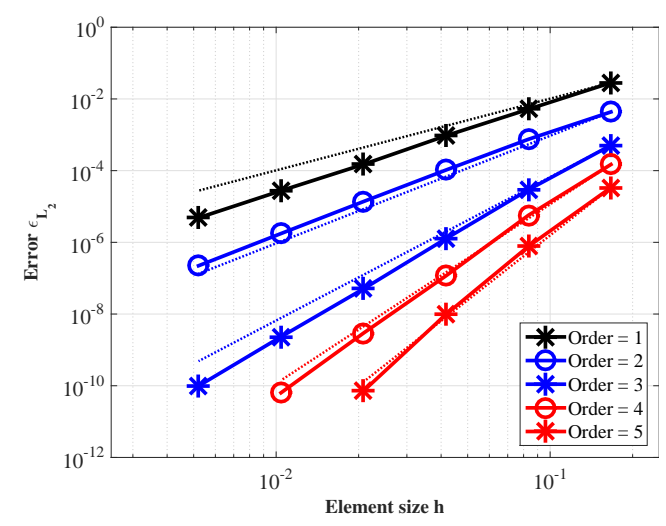

(b) Lenoir mapping

Figure 24: Approximation error measured in $L_{2}$-norm for the mappings introduced in Section 4 .

In case of unfitted interfaces, e.g. using a piecewise straight approximation of curved boundaries or material interfaces crossing the elements, the approximation quality is significantly degraded. The convergence rates are then independent of the ansatz order of the finite element functions and are limited by the smoothness of the approximated function or the accuracy of the geometry description.

The problem statement is an infinite domain consisting of material $A$ with a circular inclusion of material $B$. As before, two different background meshes are chosen, both are depicted in Fig 25 (a) and Fig 25.(b). The circle with the radius $a=0.4$ is centered in the origin. On the entire outer domain boundary, represented by red circles in in Fig 25 . Dirichlet boundary conditions are imposed using the analytical solution given in Eqs. (14) and (15).

The solution for the displacement components $u_{x}$ and $u_{y}$ in polar coordinates is given by [52, 22] as

$$
\begin{array}{ll}
u_{r}(r, \theta)= \begin{cases}\left(1-\frac{b^{2}}{a^{2}}\right) \alpha+\frac{b^{2}}{a^{2}} r, & 0 \leq r \leq a \\
\left(r-\frac{b^{2}}{r}\right) \alpha+\frac{b^{2}}{r}, & a \leq r \leq b,\end{cases} \\
u_{\theta}(r, \theta)=0
\end{array}
$$

with the parameter $\alpha$ defined as

$$
\alpha=\frac{\left(\lambda_{1}+\mu_{1}+\mu_{2}\right) b^{2}}{\left(\lambda_{2}+\mu_{2)} a^{2}+\left(\lambda_{1}+\mu_{1}\right)\left(b^{2}-a^{2}\right)+\mu_{2} b^{2}\right.} .
$$

$(\theta, r)$ are the standard polar coordinates, $t_{x}$ the traction in $x$-direction, and $\mu$ the shear modulus. For our example, plane strain conditions are considered, therefore the Kolosov constant is defined as $\kappa=3-4 \nu$, with $\nu$ being the Poisson ratio. For the numerical computations, the material constants for material $A$ and material $B$ are chosen as $\left\{E_{1}=10, \nu_{1}=0.3\right\}$ and $\left\{E_{2}=1, \nu_{2}=0.25\right\}$, respectively. For all following examples, the mesh is sequentially subdivided into $\ell_{h}=\{8,16,32,64,128\}$ quadrilateral elements per side. The characteristic element length is given as $h=1 / \ell_{h}$. A standard displacement-based finite element formulation is used to solve the equations.

The first convergence study measures the displacement error in the $L_{2}$-norm, $\varepsilon_{L_{2}}$, as introduced in Eq. (12). The dotted lines show the theoretically optimal convergence rates. Fig. 26 displays the $L_{2}$-error as a function of the characteristic element size $h$. The results validate the proposed approach and show excellent agreement between the optimal and the calculated convergence rates.

Also the error in strain energy is calculated using the analytical solution as presented in [52, 22]. The results in Fig. 27 clearly show optimal convergence rates also for the strain energy norm.

Investigating the condition number $\kappa$ of the system stiffness matrix and plotting $\kappa$ over the characteristic element length $h$, Fig. 28.a) is obtained for the Cartesian background mesh and Fig. 28.b) for the deformed background mesh. 


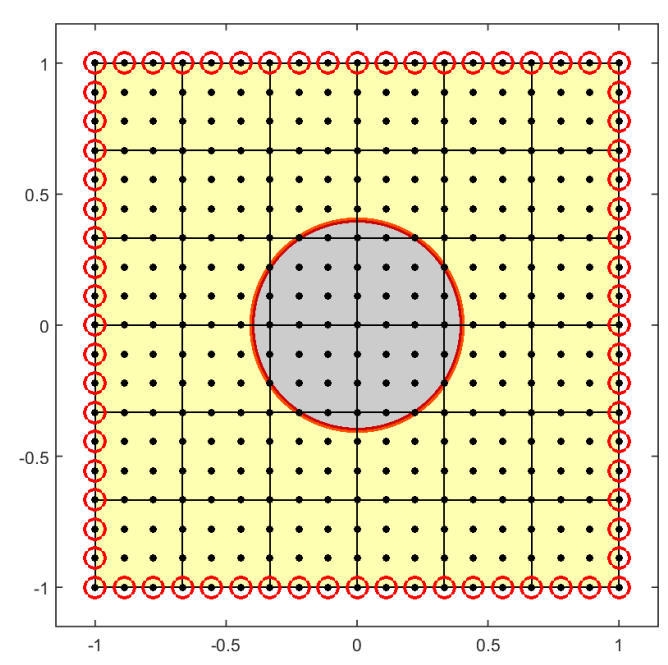

(a) Cartesian background mesh

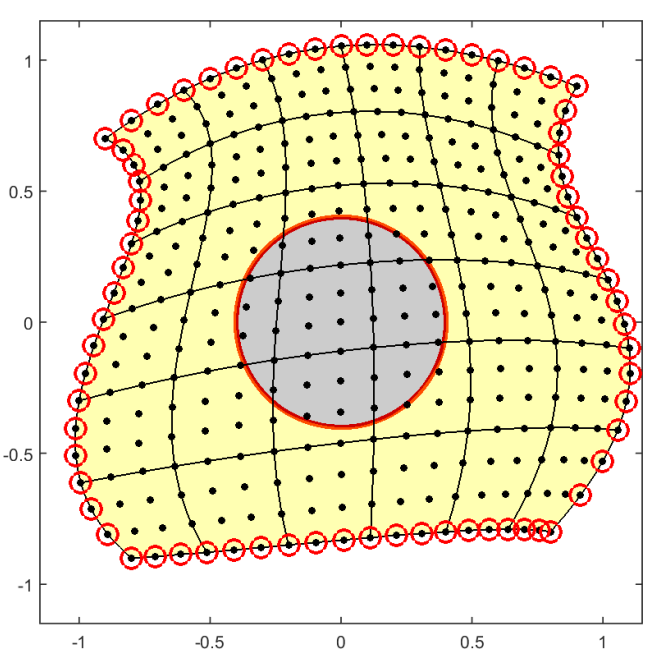

(b) Deformed background mesh

Figure 25: Background meshes for the bi-material test case. The outer element edges conform with the boundary. The interface between the materials however, is within the elements.

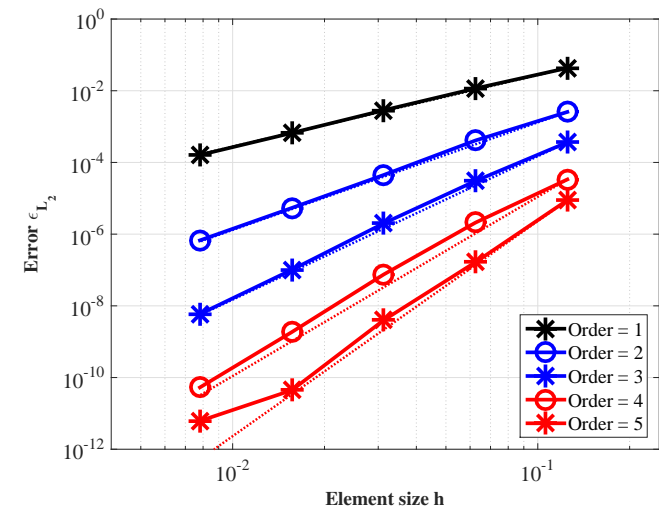

(a) Cartesian background mesh

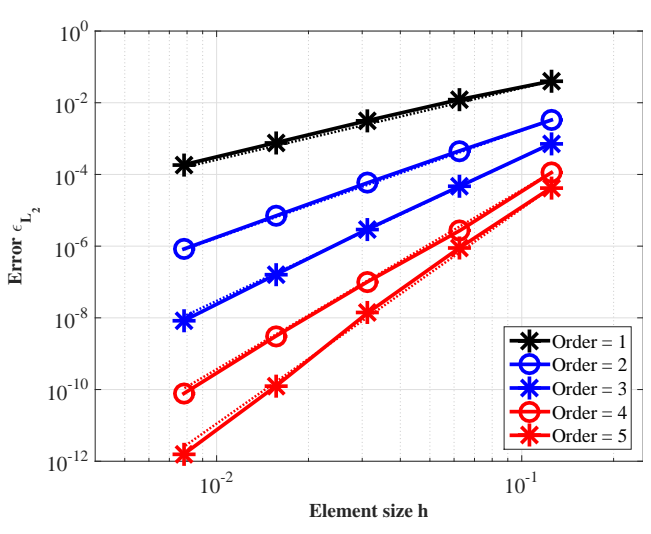

(b) Deformed background mesh

Figure 26: Convergence results for the bi-material problem with the error measured in the $L_{2}$-norm. 


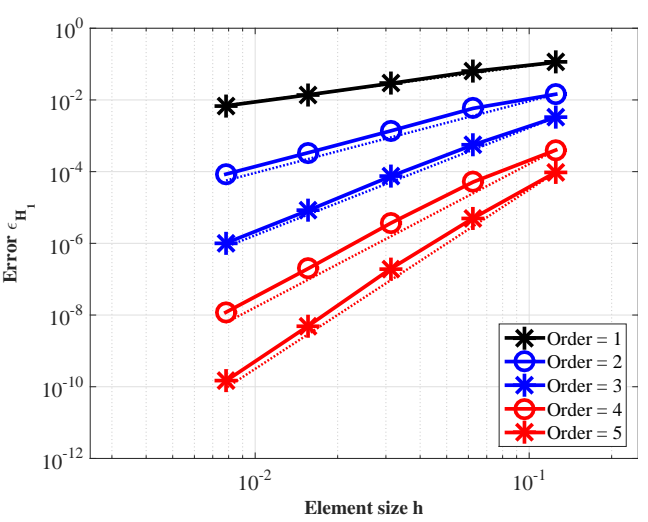

(a) Cartesian background mesh

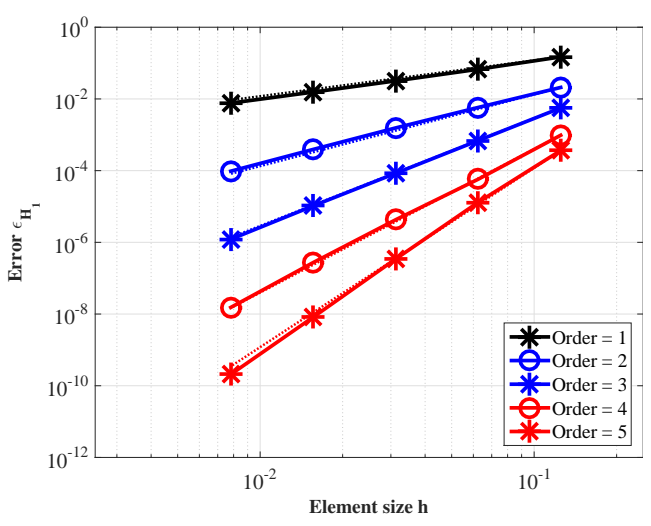

(b) Deformed background mesh

Figure 27: Convergence results for the bi-material problem with the error measured in the $H_{E}$-norm.

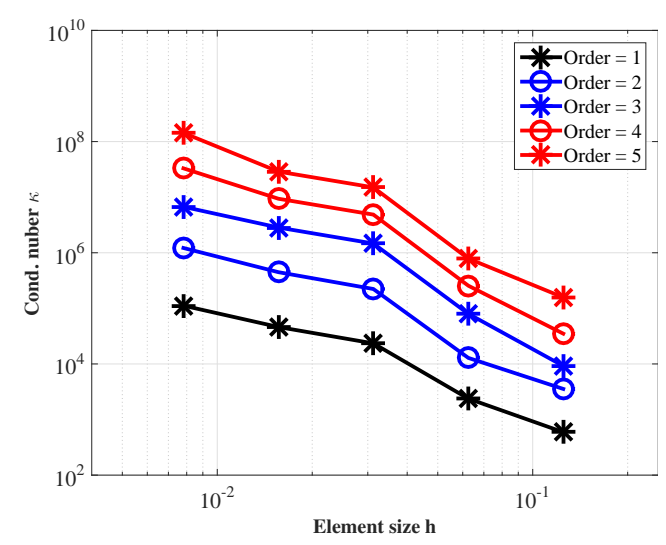

(a) Cartesian background mesh

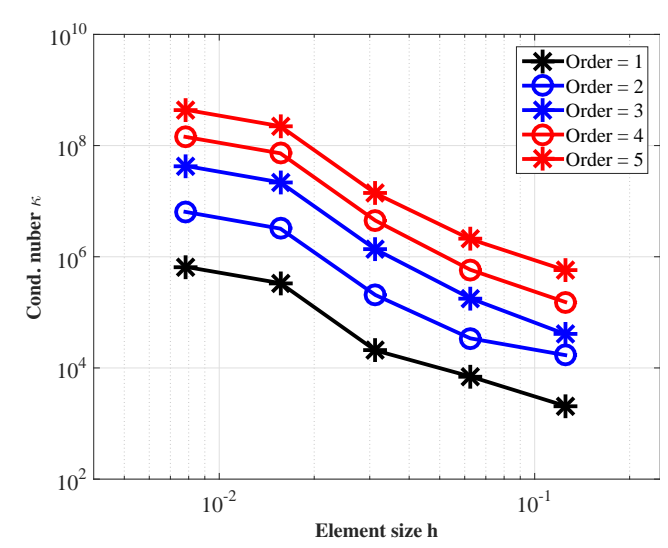

(b) Deformed background mesh

Figure 28: Dependency of the condition number on the element size for different element orders.

It is seen that in this example, the condition number $\kappa$ is not excessively high, therefore, no effort was made to manipulate the node positions in the background mesh.

\subsection{Infinite plate with a circular hole}

The last example is chosen to illustrate the performance of the proposed higher-order CDFEM in the context of fictitious domain methods where the (curved) domain boundary is described implicitly using the level set function. The example is, as before, a benchmark problem for higher order finite element procedures, see e.g. [13], [17]. The error in the displacements (measured in a $L_{2}$-norm), the error in displacement derivatives (measured in $H_{E}$-norm) and also the condition number of the equation system are investigated.

The problem statement is an infinite domain with a circular inclusion and unidirectional tension in $x$-direction. As before, two different background meshes are chosen, a Cartesian background mesh and a deformed background mesh, both are depicted in Fig. 29.a) and (b). The circular hole with the radius $a=0.4$ is centered in the origin. Instead of traction boundary conditions, on the entire outer domain boundary, represented by red circles in Fig 29. Dirichlet boundary conditions are imposed using the analytical solution given in Eq. (16) and Eq. (17). 


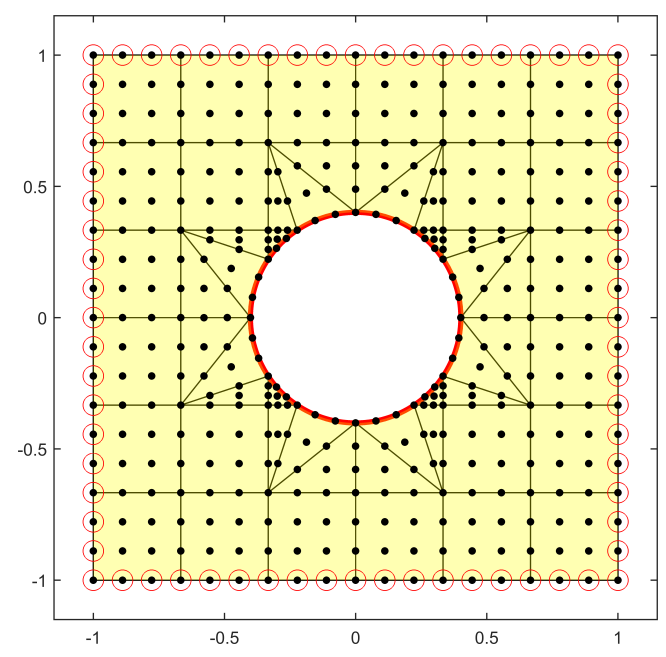

(a) Cartesian background mesh

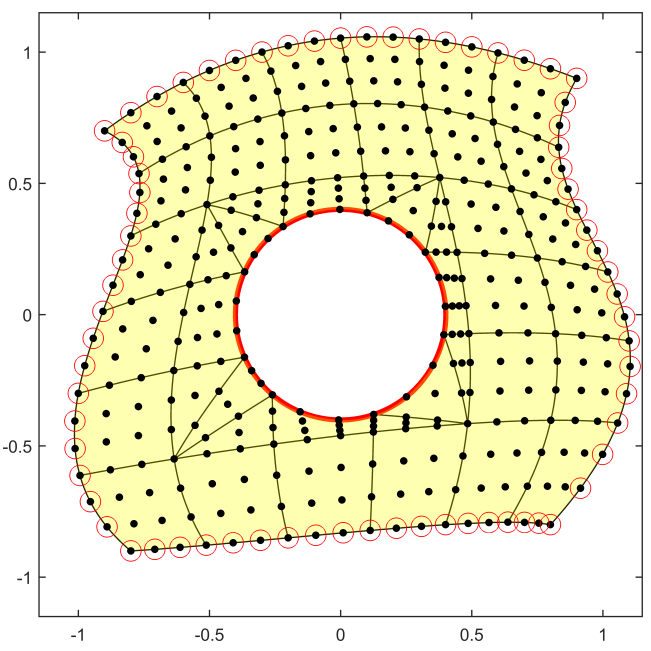

(b) Deformed mesh

Figure 29: Structured (a) and unstructured mesh (b) with Dirichlet boundary conditions.

The solution for the displacement components $u_{x}$ and $u_{y}$ in Cartesian coordinates is given in [53] as

$$
\begin{aligned}
& u_{x}(r, \theta)=\frac{t_{x} a}{8 \mu}\left[\frac{r}{a}(\kappa+1) \cos \theta+\frac{2 a}{r}((1+\kappa) \cos \theta+\cos 3 \theta)-\frac{2 a^{3}}{r^{3}} \cos 3 \theta\right] \\
& u_{y}(r, \theta)=\frac{t_{x} a}{8 \mu}\left[\frac{r}{a}(\kappa-3) \sin \theta+\frac{2 a}{r}((1-\kappa) \sin \theta+\sin 3 \theta)-\frac{2 a^{3}}{r^{3}} \sin 3 \theta\right]
\end{aligned}
$$

with $(\theta, r)$ being the standard polar coordinates, $t_{x}$ the traction in $x$-direction, and $\mu$ the shear modulus. For our example, plane strain conditions are considered, therefore the Kolosov constant is given as $\kappa=3-4 \nu$, with $\nu$ as Poisson ratio. For the following numerical computations, the material constants are chosen as $E=10^{4}$ and $\nu=0.3$.

As before, the mesh is sequentially subdivided in $\ell_{h}=\{8,16,32,64,128\}$ quadrilateral elements per side. The automatically generated elements inside the inclusion are simply neglected when integrating the weak form of the partial differential equation. The first convergence study measures the displacement error in $L_{2}$-norm, $\varepsilon_{L_{2}}$ as introduced in Eq. (12). The dotted lines show the theoretically optimal convergence rates. Fig. 30 displays the error as a function of the characteristic element length $h$. The results show again excellent agreement between the optimal and the calculated convergence rates.

The exact stresses and strains are given in e.g. [13], [17]. The error in strain energy is calculated using the error measure in Eq. (13). The results are shown in Fig. 31 and are again optimal. The condition number for the Cartesian and the deformed background mesh are plotted in Fig. 32,a) and Fig. 32.(b).

\section{Conclusions, Limitations and Perspectives}

In this work, a higher-order conformal decomposition finite element method (CDFEM) has been proposed. It combines the advantages of the FEM on body fitted meshes (application of boundary and interface conditions, numerical integration) with those of embedded domain methods (no explicit manual mesh generation). The key-aspect is the automatic decomposition of cut background elements into higher-order conforming sub-elements.

Therefore, a two-step procedure is suggested: In the first part, the implicit interfaces described by the level set method are detected and meshed. Then, based on the topological situation in the cut element, higher-order elements are mapped on the two sides of the interface. It is shown that the location of the inner element nodes, depending on the concrete mapping, have important implications for the achieved convergence rates.

The reconstruction and remeshing scheme is executed in the reference element, therefore also non-structured background meshes can be used. Under restrictions on the curvature of the level set function, all topological situations 


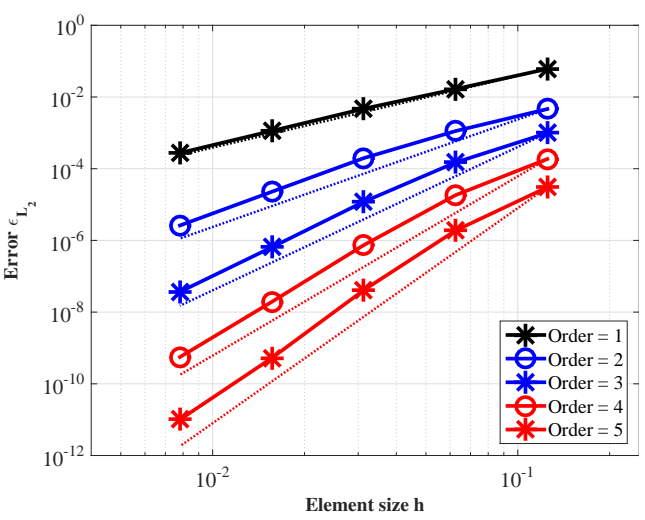

(a) Cartesian background mesh

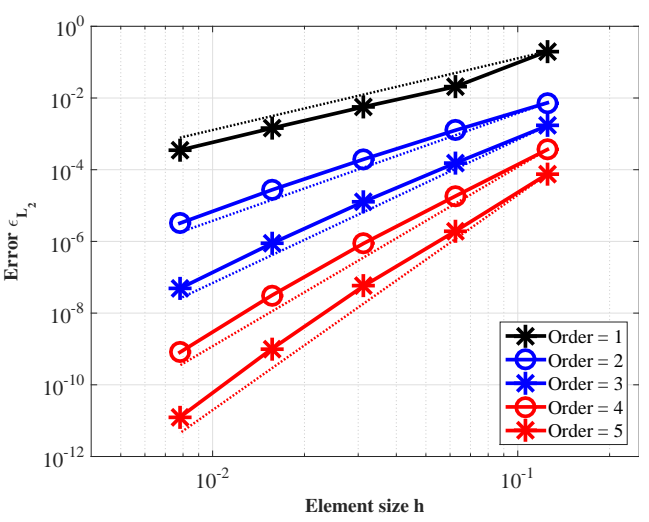

(b) Deformed background mesh

Figure 30: Convergence study for the displacements in the $L_{2}$-norm, Eq. 12.

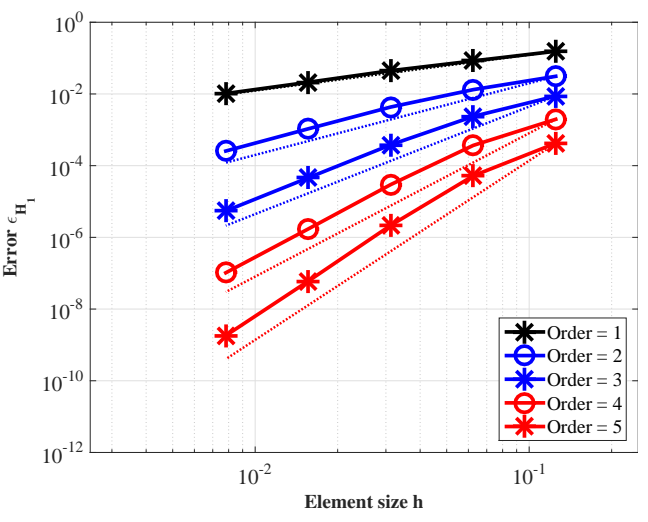

(a) Cartesian background mesh

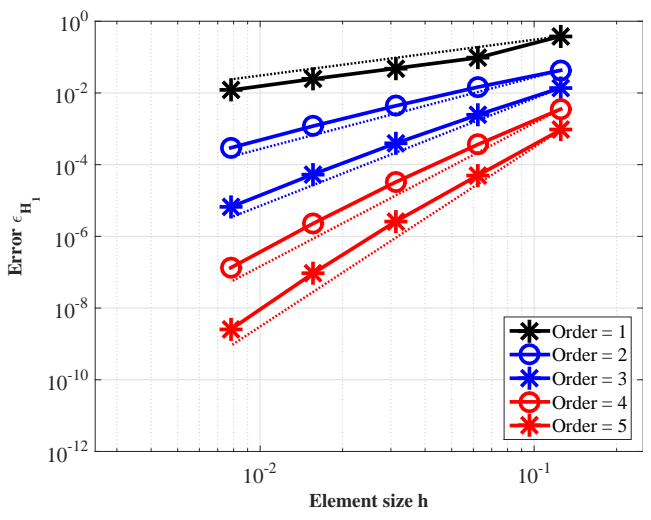

(b) Deformed background mesh

Figure 31: Convergence results for the plate with a circular hole problem in the $H_{E}$-norm.

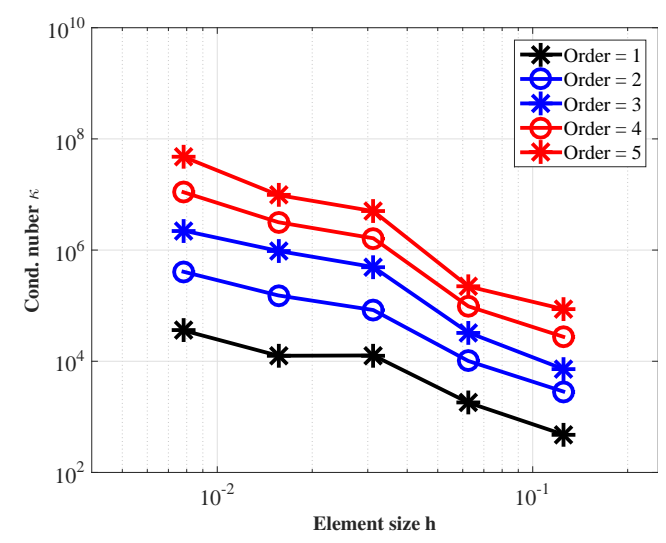

(a) Cartesian background mesh

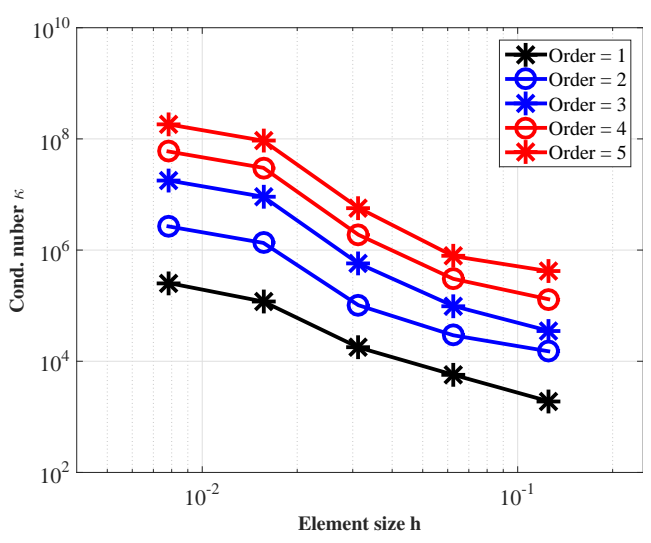

(b) Deformed background mesh

Figure 32: Dependency of the condition number on the element size for different element orders. 
can be covered. Used in a FEM simulation, the automatically determined sub-elements may lead to an ill-conditioning of the system matrix. Several solution strategies will be investigated in future works. Herein, none of these techniques have been employed and, nevertheless, excellent have been obtained with direct solvers.

The proposed approach shows excellent agreement between optimal and calculated convergence rates. It is very flexible with respect to the employed element types and not limited to structured background meshes. It shows a stable behaviour even for geometrically complex interfaces. The method is able to automatically remesh a broad class of problems with an implicitly sharp interface representation. A number of extensions for the method can be conceived, for example, evolving interfaces that occur in plasticity or two-phase flows. Also an extension to three-dimensional problems is currently in progress. It is also planned to combine the approach with adaptive refinements such that even more complex geometrical situations can be covered automatically.

\section{References}

[1] I. Babuška. The finite element method for elliptic equations with discontinuous coefficients. Computing, 5(3):207-213, 1970.

[2] I. Babuška, U. Banerjee, and J.E. Osborn. Meshless and generalized finite element methods: A survey of some major results. In M. Griebel and M.A. Schweitzer, editors, Meshfree Methods for Partial Differential Equations, volume 26 of Lectures Notes in Computational Science and Engineering. Springer, Berlin, 2002.

[3] I. Babuška, U. Banerjee, and J.E. Osborn. Generalized finite element methods: Main ideas, results and perspectives. Int. J. Comp. Meth., $1: 67-103,2004$

[4] P. Bastian and C. Engwer. An unfitted finite element method using discontinuous galerkin. Internat. J. Numer. Methods Engrg., 79(12):15571576,2009

[5] T. Belytschko and T. Black. Elastic crack growth in finite elements with minimal remeshing. Internat. J. Numer. Methods Engrg., 45:601 620, 1999.

[6] T. Belytschko, C. Parimi, N. Moës, N. Sukumar, and S. Usui. Structured extended finite element methods for solids defined by implicit surfaces. Internat. J. Numer. Methods Engrg., 56:609 - 635, 2003.

[7] T. Belytschko, S.P. Xiao, and C. Parimi. Topology optimization with implicit functions and regularization. Internat. J. Numer. Methods Engrg., 57:1177 - 1196, 2003.

[8] C. Bernardi. Optimal finite-element interpolation on curved domains. SIAM Journal on Numerical Analysis, 26(5):1212-1240, 1989.

[9] E. Burman, S. Claus, P. Hansbo, M.G. Larson, and A. Massing. CutFEM: Discretizing geometry and partial differential equations. Internat. J. Numer. Methods Engrg., 2014.

[10] E. Burman and P. Hansbo. Fictitious domain finite element methods using cut elements: I. a stabilized Lagrange multiplier method. Comp. Methods Appl. Mech. Engrg., pages 2680 - 2686, 2010.

[11] E. Burman and P. Hansbo. Fictitious domain finite element methods using cut elements: II. A stabilized Nitsche method. Applied Numerical Mathematics, 62:328 - 341, 2012

[12] A. Byfut, A. Schröder, and C. Carstensen. $h p$ - adaptive extended finite element method. In T.P. Fries and A. Zilian, editors, Proceedings of the ECCOMAS Thematic Conference XFEM 2009, Aachen, Germany, 2009.

[13] K.W. Cheng and T.P. Fries. Higher-order XFEM for curved strong and weak discontinuities. Internat. J. Numer. Methods Engrg., 82:564 $590,2010$.

[14] J. Chessa, P. Smolinski, and T. Belytschko. The extended finite element method (XFEM) for solidification problems. Internat. J. Numer. Methods Engrg., 53:1959 - 1977, 2002.

[15] P.G. Ciarlet and P.-A. Raviart. Interpolation theory over curved elements, with applications to finite element methods. Computer Methods in Applied Mechanics and Engineering, 1(2):217 - 249, 1972.

[16] G.H. Cottet and E. Maitre. A level-set formulation of immersed boundary methods for fluid-structure interaction problems. Comptes Rendus Mathematique, 338:581 - 586, 2004.

[17] C. Daux, N. Moës, J. Dolbow, N. Sukumar, and T. Belytschko. Arbitrary branched and intersecting cracks with the extended finite element method. Internat. J. Numer. Methods Engrg., 48:1741 - 1760, 2000.

[18] A. Düster, Z. Yang J. Parvizian, and E. Rank. The finite cell method for three-dimensional problems of solid mechanics. Comp. Methods Appl. Mech. Engrg., 197:3768 - 3782, 2008

[19] K. J. Fidkowski and D. L. Darmofal. A triangular cut-cell adaptive method for high-order discretizations of the compressible navier-stokes equations. J. Comput. Phys., 225(2):1653-1672, August 2007.

[20] S. Frei and T. Richter. A locally modified parametric finite element method for interface problems. SIAM Journal on Numerical Analysis, 52(5):2315-2334, 2014.

[21] T.-P. Fries and S. Omerović. Higher-order accurate integration of implicit geometries. Internat. J. Numer. Methods Engrg., pages n/a-n/a, 2015. nme.5121.

[22] T.P. Fries and T. Belytschko. The intrinsic XFEM: A method for arbitrary discontinuities without additional unknowns. Internat. J. Numer. Methods Engrg., 68:1358 - 1385, 2006.

[23] T.P. Fries and T. Belytschko. The extended/generalized finite element method: An overview of the method and its applications. Internat. J. Numer. Methods Engrg., 84:253 - 304, 2010.

[24] R. Glowinski, T.W. Pan, T.I. Hesla, D.D. Joseph, and J. Périaux. A distributed Lagrange multiplier/fictitious domain method for flows around moving rigid bodies: Application to particulate flow. Int. J. Numer. Methods Fluids, 30:1043 - 1066, 1999.

[25] W.J. Gordon and C.A. Hall. Construction of curvi-linear co-ordinate systems and applications to mesh generation. Internat. J. Numer. Methods Engrg., 7:461 - 477, 1973. 
[26] W.J. Gordon and C.A. Hall. Transfinite element methods: blending function interpolation over arbitrary curved element domains. Numer. Math., 21:109 - 129, 1973.

[27] A. Hansbo and P. Hansbo. An unfitted finite element method, based on Nitsche's method, for elliptic interface problems. Comp. Methods Appl. Mech. Engrg., 191:5537 - 5552, 2002.

[28] R. M. J. Kramer and D. R. Noble. A conformal decomposition finite element method for arbitrary discontinuities on moving interfaces. Internat. J. Numer. Methods Engrg., 100(2):87-110, 2014.

[29] F. Labelle and J. Shewchuk. Isosurface stuffing: Fast tetrahedral meshes with good dihedral angles. ACM Transactions on Graphics, 26:57.1 $-57.10,2007$.

[30] G. Legrain. A nurbs enhanced extended finite element approach for unfitted cad analysis. Comput. Mech., 52(4):913-929, 2013.

[31] C. Lehrenfeld. High order unfitted finite element methods on level set domains using isoparametric mappings. Comp. Methods Appl. Mech. Engrg., 300:716 - 733, 2016.

[32] M. Lenoir. Optimal isoparametric finite elements and error estimates for domains involving curved boundaries. Society of Industrial and Applied Mathematics, 23:562 - 580, 1986.

[33] Z. Li. A fast iterative algorithm for elliptic interface problems. SIAM J. Numer. Anal., 35(1):230-254, 1998.

[34] S. Loehnert. A stabilization technique for the regularization of nearly singular extended finite elements. Comput. Mech., 54(2):523-533, 2014.

[35] Q. Lu, M. S. Shephard, S. Tendulkar, and M. W. Beall. Parallel mesh adaptation for high-order finite element methods with curved element geometry. Engineering with Computers, 30(2):271-286, 2014

[36] X.-J. Luo, M. S. Shephard, R. O’Bara, R. Nastasia, and M. W. Beall. Automatic p-version mesh generation for curved domains. Engineering with Computers, 20(3):273-285, 2004

[37] N. Moës, M. Cloirec, P. Cartraud, and J.F. Remacle. A computational approach to handle complex microstructure geometries. Comp. Methods Appl. Mech. Engrg., 192:3163-3177, 2003.

[38] N. Moës, J. Dolbow, and T. Belytschko. A finite element method for crack growth without remeshing. Internat. J. Numer. Methods Engrg., 46:131 - 150, 1999.

[39] D. R. Noble, E. P. Newren, and J. B. Lechman. A conformal decomposition finite element method for modeling stationary fluid interface problems. Int. J. Numer. Methods Fluids, 63(6):725-742, 2010.

[40] S. Osher and R.P. Fedkiw. Level set methods: an overview and some recent results. J. Comput. Phys., 169:463 - 502, 2001.

[41] S. Osher and R.P. Fedkiw. Level Set Methods and Dynamic Implicit Surfaces. Springer, Berlin, 2003.

[42] J. Parvizian, A. Düster, and E. Rank. Finite cell method : h- and p-extension for embedded domain problems in solid mechanics. Comput. Mech., 41:121 - 133, 2007.

[43] C. S. Peskin. Flow patterns around heart valves: a digital computer method for solving the equations of motion. Phd thesis, Albert Einstein College of Medicine, Yeshiva University, 1972.

[44] R. Rangarajan and A. Lew. Universal meshes: A method for triangulating planar curved domains immersed in nonconforming meshes. Internat. J. Numer. Methods Engrg., 98(4):236-264, 2014.

[45] D. Schillinger, A. Düster, and E. Rank. The hp-d-adaptive finite cell method for geometrically nonlinear problems of solid mechanics. Internat. J. Numer. Methods Engrg., 89:1171 - 1202, 2012

[46] D. Schillinger, M. Ruess, N. Zander, Y. Bazilevs, A. Düster, and E. Rank. Small and large deformation analysis with the p- and B-spline versions of the Finite Cell Method. Comput. Mech., 50:445 - 478, 2012.

[47] L. R. Scott. Finite element techniques for curved boundaries. PhD thesis, Massachusetts Institute of Technology, 1973.

[48] M. S. Shephard, J. E. Flaherty, K. E. Jansen, X. Li, X. Luo, N. Chevaugeon, J.-F. Remacle, M. W. Beall, and R. M. O'Bara. Adaptive mesh generation for curved domains. Applied Numerical Mathematics, 52(23):251 - 271, 2005. \{ADAPT\} '03: Conference on Adaptive Methods for Partial Differential Equations and Large-Scale Computation.

[49] S. J. Sherwin and J. Peiró. Mesh generation in curvilinear domains using high-order elements. Internat. J. Numer. Methods Engrg., 53(1):207223, 2002.

[50] P. Šolín, K. Segeth, and I. Doležel. Higher-order finite element methods. CRC Press, Boca Raton, FL, 2003.

[51] G. Strang and G. Fix. An Analysis of the Finite Element Method. Wellesley-Cambridge Press, 2008.

[52] N. Sukumar, D.L. Chopp, N. Moës, and T. Belytschko. Modeling holes and inclusions by level sets in the extended finite-element method. Comp. Methods Appl. Mech. Engrg., 190:6183 - 6200, 2001.

[53] B. Szabó and I. Babuška. Finite Element Analysis. John Wiley \& Sons, Chichester, 1991.

[54] B. Szabó, A. Düster, and E. Rank. The p-Version of the Finite Element Method, chapter 5, pages 119 - 139. John Wiley \& Sons, Chichester, 2004.

[55] J. A. Trangenstein. Numerical Solution of Elliptic and Parabolic Partial Differential Equations. Cambridge University Press, 2013. Cambridge Books Online.

[56] X. Wang, M.Y. Wang, and D. Guo. Structural shape and topology optimization in a level-set-based framework of region representation. Structural and Multidisciplinary Optimization, 27:1 - 19, 2004. 